\title{
Absolute Stability of Wavetrains Can Explain Spatiotemporal Dynamics in Reaction-Diffusion Systems of Lambda-Omega Type*
}

\author{
Matthew J. Smith ${ }^{\dagger}$, Jens D. M. Rademacher ${ }^{\ddagger}$, and Jonathan A. Sherratt ${ }^{\S}$
}

\begin{abstract}
The lambda-omega class of reaction-diffusion equations has received considerable attention because they are more amenable to mathematical investigation than other oscillatory reaction-diffusion systems and include the normal form of any reaction-diffusion system with scalar diffusion close to a standard supercritical Hopf bifurcation. Despite this, detailed studies of the dynamics predicted by numerical simulations have mostly been restricted to regions of parameter space in which stable wavetrains (periodic traveling waves) are selected by the initial or boundary conditions; we use the term "stability" to denote spectral stability on the real line. Here we consider the emergent spatiotemporal dynamics on large bounded domains, with Dirichlet conditions at one boundary and Neumann conditions at the other. Previous studies have established a parameter threshold below which stable wavetrains are generated by the Dirichlet boundary condition. We use numerical continuation techniques to analyze the spectral stability of wavetrain solutions, and we identify a second stability threshold, above which the selected wavetrain is absolutely unstable. In addition, we prove that the onset of absolute stability always occurs through a complex conjugate pair of branch points in the absolute spectrum, which greatly simplifies the detection of this threshold. In the parameter region in which the spectra of the selected waves indicate instability but absolute stability, our numerical simulations predict so-called "source-sink" dynamics: bands of visibly regular periodic traveling waves that are separated by localized defects. Beyond the absolute stability threshold our simulations predict irregular spatiotemporal behavior.
\end{abstract}

Key words. AUTO, parabolic partial differential equations, complex Ginzburg-Landau equation, absolute spectrum, plane waves

AMS subject classifications. $35 \mathrm{~K} 57,35 \mathrm{~B} 35,35 \mathrm{P} 05$

DOI. $10.1137 / 090747865$

1. Introduction. A wide range of physical and natural systems exhibit dynamics that are the result of local interactions between their components and of diffusive dispersal. The dynamics of such systems are often studied mathematically using reaction-diffusion equations. An important subset of such equations are those in which the local interactions between the components of the system lead to cyclic dynamics, so that the reaction kinetics have a stable limit cycle. Such oscillatory reaction-diffusion systems have been used effectively as models

\footnotetext{
* Received by the editors January 26, 2009; accepted for publication (in revised form) by D. Barkley June 19, 2009; published electronically August 26, 2009.

http://www.siam.org/journals/siads/8-3/74786.html

${ }^{\dagger}$ Computational Ecology and Environmental Science Group, Microsoft Research, Cambridge CB3 OFB, UK (Matthew.Smith@Microsoft.com). This author was supported in part by an Environmental Mathematics and Statistics (EMS) studentship from the Natural Environment Research Council (NERC) and the Engineering and Physical Sciences Research Council (EPSRC), and by Microsoft Research.

${ }^{\ddagger}$ Centre for Mathematics and Computer Science (CWI), Kruislaan 413, 1098 SJ Amsterdam, The Netherlands (rademach@cwi.nl). The work of this author was supported by the NWO cluster NDNS+.

${ }^{\S}$ Department of Mathematics and Maxwell Institute for Mathematical Sciences, Heriot-Watt University, Edinburgh EH14 4AS, UK (jas@ma.hw.ac.uk). This author was supported in part by a Leverhulme Trust Research Fellowship.
} 
for many phenomena (see [26] for a review). Oscillatory reaction-diffusion equations can generate a wide range of spatiotemporal dynamics, including target patterns, spiral and scroll waves, localized breathers, and spatiotemporal irregularities (see [14, 58, 35] for reviews of these phenomena). From an applied perspective, studies of such equations center around understanding the predicted dynamics and interpreting their implications for the physical, chemical, or biological systems concerned. A key issue for this is the stability of spatiotemporal solutions. The building block for all such solutions is one-dimensional wavetrains, and their spectral stability on unbounded and large bounded domains (see [35] for a review) is the focus of this paper.

Over the last decade, significant new mathematical insights have been gained into the stability of solutions of reaction-diffusion equations. In particular, it is now clear that the understanding of spatiotemporal behavior is helped by considering various different types of (in)stability and by distinguishing stability on the real line and on large periodic domains from stability on large bounded domains with separated boundary conditions (see [38] for a more detailed discussion than that given here). These distinctions are made on the level of the spectrum of the equations when linearized about a solution, and we call a solution (spectrally) stable if, on the whole real line, all modes (except the neutral translation mode) decay on the real line. Classifications of instability distinguish between different spatiotemporal dynamics of the growing perturbations. On the real line, for "convectively unstable" solutions, perturbations grow while traveling away from the site of the perturbation but decay at the site of perturbation itself; by contrast, for "absolutely unstable" solutions, perturbations grow at all points in space, including the site of perturbation. The most widespread application of these different types of stability has been to problems in fluid dynamics (reviewed by [21, 13]) and spiral wave break-up (e.g., [2, 39, 59]).

In this paper we study the stability of wavetrain solutions for a particular system of oscillatory reaction-diffusion equations in one space dimension:

$$
\begin{aligned}
& u_{t}=u_{x x}+\left(1-r^{2}\right) u-\left(\omega_{0}-\omega_{1} r^{2}\right) v, \\
& v_{t}=v_{x x}+\left(1-r^{2}\right) v+\left(\omega_{0}-\omega_{1} r^{2}\right) u .
\end{aligned}
$$

Here $u$ and $v$ are the variables of the system, subscripts $t$ and $x$ denote partial derivatives with respect to time and space, $r=\left(u^{2}+v^{2}\right)^{1 / 2}$, and $\omega_{0}$ and $\omega_{1}$ are constants. For mathematical simplicity, we will assume that $\omega_{0}>\omega_{1}>0$, which ensures that $\omega_{0}-\omega_{1} r^{2} \neq 0$ for any $r \in[0,1]$, which is an invariant region [6]. Equations (1) are a special case of the complex GinzburgLandau equation (see [1] for a review) and have a wide relevance for oscillatory systems, being the normal form of an oscillatory reaction-diffusion system with equal diffusivities, when the kinetics are close to a standard supercritical Hopf bifurcation. They belong to the "lambdaomega" class of reaction-diffusion equations, introduced by Kopell and Howard [22]. These authors showed that such equations are much more amenable to mathematical analysis than most oscillatory reaction-diffusion equations. In particular, they showed that (1) has a oneparameter family of wavetrain solutions, of the form

$$
\begin{aligned}
& u=R \cos \left[\theta_{0} \pm x \sqrt{1-R^{2}}+\left(\omega_{0}-\omega_{1} R^{2}\right) t\right], \\
& v=R \sin \left[\theta_{0} \pm x \sqrt{1-R^{2}}+\left(\omega_{0}-\omega_{1} R^{2}\right) t\right],
\end{aligned}
$$

Copyright (c) by SIAM. Unauthorized reproduction of this article is prohibited. 
where amplitude $R(0 \leq R \leq 1)$ parameterizes the family and $\theta_{0}$ is an arbitrary constant. Moreover, they calculated an exact condition for the stability of the waves, namely,

$$
R>r_{\text {ess }}=\left\{\frac{2+2 \omega_{1}^{2}}{3+2 \omega_{1}^{2}}\right\}^{1 / 2} .
$$

Simulation-based studies of oscillatory reaction-diffusion equations have shown that wavetrains often arise when initial or boundary conditions are inconsistent with spatially uniform oscillations. Further, the relative simplicity of (1) has enabled such "wave selection" problems to be studied analytically [44, 42, 18, 41, 50]. One important conclusion in these papers is that for some parameters, the selected wave is in fact unstable. In such cases, numerical simulations often show spatiotemporal irregularity as the long-term behavior. However, no detailed characterization of this behavior has previously been undertaken. Our objective in this paper is to obtain a deeper understanding of the spatiotemporal behavior arising in simulations of (1) in the regions of parameter space in which unstable waves are selected. We will principally focus on wave selection in (1) by Dirichlet boundary conditions at one edge of a large bounded domain. We have used this wave selection scenario in the past to investigate the occurrence of wavetrains in ecological systems, where the Dirichlet boundary condition is analogous to an inhospitable environment at the edge of a habitat (see [47] for a recent review), and it has also been used to investigate the generation of traveling waves in oscillatory chemical reactions [3]. Our study here is intended to benefit future research in these areas by illustrating how the variety of dynamics observed in numerical simulations can be explained by analyzing the spectral stability of the selected wavetrains. In the discussion (section 6) we also briefly consider the generation of wavetrains when the spatially uniform (unstable) steady state $u=v=0$ receives a small localized perturbation. This "invasion" scenario has been studied extensively in the context of physical, chemical, and ecological systems $[31,57,19,47,48]$.

In our study here we will determine which of the unstable waves are absolutely unstable, using the numerical continuation approach developed by [33]. We will show that this spectral information alone gives valuable insights into the spatiotemporal dynamics resulting from solutions of the partial differential equations. We begin (section 2) by describing the results of numerical simulations of wavetrain generation. We then (section 3) outline the general theory of absolute stability and prove a new result on the relationship between the original "pinching condition" for absolute stability [12] and the more recent approach using the absolute spectrum [38]. We also prove that for the particular case of the lambda-omega system (1), absolute stability is determined by specific points in the absolute spectrum, known as "branch points" [38]. In section 4 we develop a systematic approach for the numerical calculation of the absolute spectrum. This provides an efficient way of identifying parameter thresholds for absolute stability, and the supplementary online material [49] contains a detailed tutorial guide and sample code to facilitate the implementation and adaptation of this approach by others. In section 5 we discuss details of the "source-sink" dynamics that we observe for parameters such that the boundary condition selects a convectively unstable wavetrain. Finally, in section 6 we draw conclusions and briefly discuss the dynamics that arise from wavetrain generation behind invasion fronts.

Copyright (c) by SIAM. Unauthorized reproduction of this article is prohibited. 

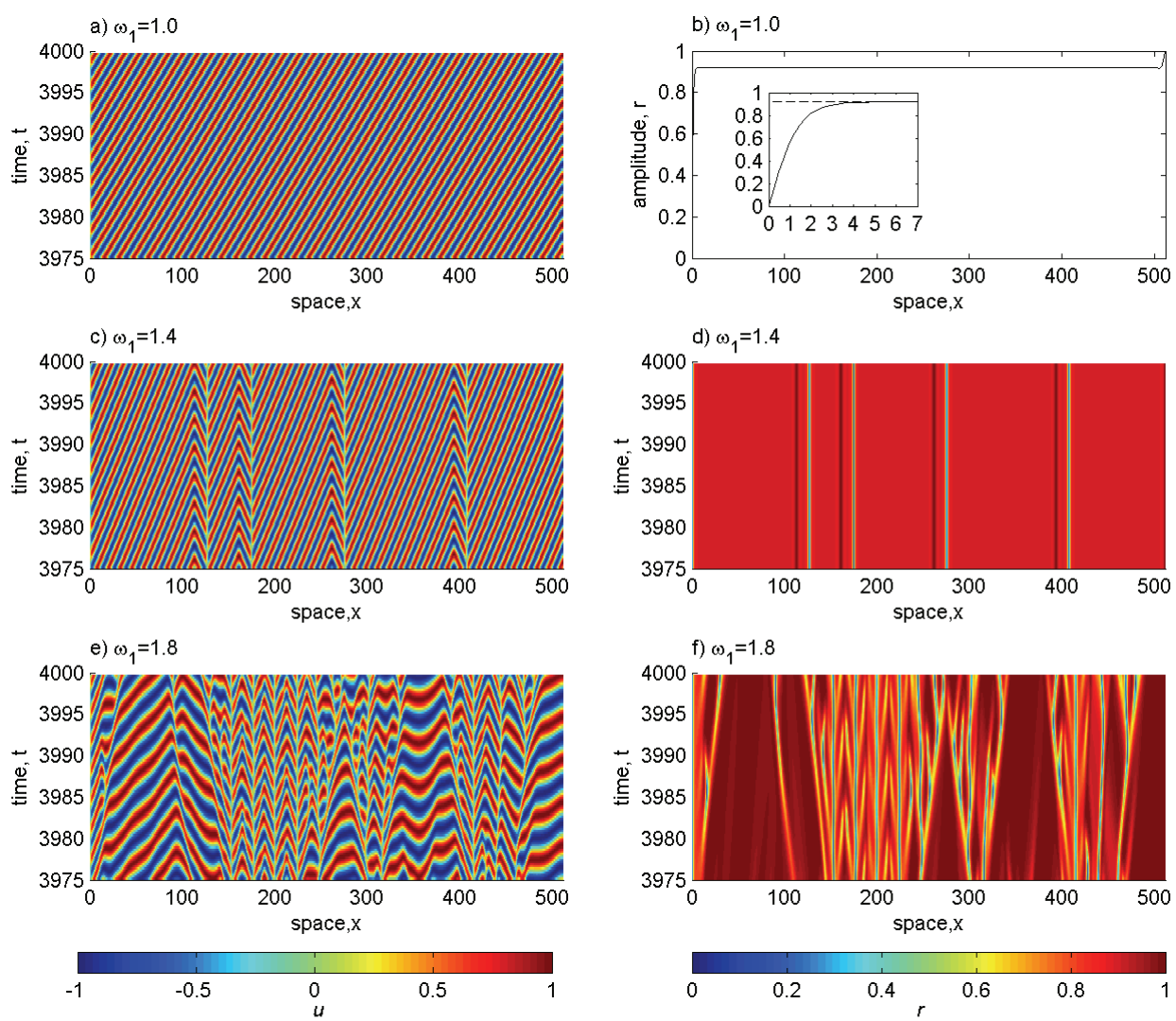

Figure 1. Example spatiotemporal dynamics for the reaction-diffusion system (1) with Dirichlet boundary conditions $u=v=0$ at $x=0$, and $u_{x}=v_{x}=0$ at the right-hand boundary $x=512$. In all cases $\omega_{0}=3.0$, and we plot $u$ and the solution amplitude $r=\sqrt{u^{2}+v^{2}}$. For clarity of presentation, we plot the long-term solution for $r$ as a function of space $x$ at one time point in (b), and show space-time plots in (a) and (c)-(f). In (a),(b) we demonstrate the selection of a stable wave $\left(\omega_{1}=1.0\right)$; the inset in (b) shows the behavior close to the $x=0$ boundary, with the dotted line indicating the analytically derived solution amplitude $r_{\text {dir }}$ given by (4). In (c),(d) and (e),(f) we show the spatiotemporal dynamics for larger values of $\omega_{1}\left(\omega_{1}=1.4\right.$ and $\omega_{1}=1.8$, respectively), which imply selection of a wavetrain that is unstable in both cases, but convectively unstable in (c),(d) and absolutely unstable in (e),(f). The equations were solved numerically using a semi-implicit finite difference method, with the grid spacing set by dividing the domain into 2101 points, and with time step $\delta t=0.001$. Note that in (c),(d) the exact location of the right-most source and sink can be sensitive to the domain length.

2. Wavetrain generation by a Dirichlet boundary condition. When (1) is solved on $0<x<L$ with $L$ large, subject to $u=v=0$ at $x=0$ and $u_{x}=v_{x}=0$ at $x=L$ and with $\omega_{1}$ such that criterion (3) is satisfied, the long-term solution away from the boundaries consists of a wavetrain (illustrated in Figure 1(a),(b)). (By "long-term" we mean that sufficient time has elapsed for the initial transient dynamics to have disappeared.) Our requirement that $\omega_{0}>\omega_{1}>0$ implies that these waves travel in the positive $x$ direction; waves moving towards the boundary do occur for other parameters (see [41] for examples). Sherratt [41] showed that the amplitude of these waves is

$$
r_{d i r} \equiv\left\{\frac{1}{2}\left[1+\sqrt{1+\frac{8}{9} \omega_{1}^{2}}\right]\right\}^{-1 / 2} .
$$

Copyright $@$ by SIAM. Unauthorized reproduction of this article is prohibited. 
Close to the $x=0$ boundary, the solution amplitude drops from this value to zero; specifically the long-term solution away from $x=L$ is (see [41])

$$
r=r_{d i r} \tanh (x / \sqrt{2}) .
$$

In the language of the complex Ginzburg-Landau equation, this solution form is a stationary Nozaki-Bekki hole [27, 29, 4]. One simple application of (4) is to assess the accuracy of numerical solutions, and throughout this study we will use a numerical discretization that gives a wave amplitude differing (in stable cases) from (4) by about $0.1 \%$. Note that the space and (especially) time step required for this depend strongly on $\omega_{0}$ (see Appendix C of [50] for details).

The criterion (3) of Kopell and Howard [22] implies that the wavetrain with amplitude $r_{d i r}$ is stable (on the real line or in the limit of large $L$ for periodic boundary conditions) if and only if $\omega_{1}<1.110468$. A key focus of our work is to calculate the corresponding condition for absolute stability, which we will show to be $\omega_{1}<1.576465$. In Figure $1(\mathrm{c})-(\mathrm{f})$, we show typical numerical simulations for one value of $\omega_{1}$ below this threshold (but above 1.110468) and one above it. Corresponding movies, which are very helpful in understanding the dynamics, can be viewed at http://research.microsoft.com/en-us/projects/loptw/, or can be generated by running the "Lambda-Omega Equations Solver" (which is freely available from the same Web page).

When a convectively unstable wave is selected, the long-term behavior consists of bands of periodic waves traveling in opposite directions and separated by apparently stationary defects (Figure 1(c),(d)). We will discuss this solution form in detail in section 5, showing that the defects do in fact move over very long time scales. In contrast, for $\omega_{1}>1.576465$, so that an absolutely unstable wave is selected, the solution is irregular in space and time (Figure $1(\mathrm{e}),(\mathrm{f}))$.

3. Absolute stability of wavetrains. To calculate spectral stability of a wavetrain solution of a reaction-diffusion system we linearize the equations about this solution. In general, the coefficients of the resulting linear equations are periodic functions of the traveling wave coordinate. However, lambda-omega equations such as (1) can be converted into amplitudephase form, and linearizing about a wavetrain then gives a system with constant coefficients. From the viewpoint of numerical continuation methods for determining stability, this is a major simplification (see [33] for a detailed discussion). Substituting $u=r \cos \theta$ and $v=r \sin \theta$ into (1) gives

$$
\begin{aligned}
& r_{t}=r_{x x}+r\left(1-r^{2}\right)-r \theta_{x}^{2}, \\
& \theta_{t}=\theta_{x x}+\left(\omega_{0}-\omega_{1} r^{2}\right)+\frac{2 r_{x} \theta_{x}}{r},
\end{aligned}
$$

with the wavetrain solution (2) being $r=R$ and $\theta=\left(\omega_{0}-\omega_{1} R^{2}\right) t \pm x \sqrt{1-R^{2}}(0 \leq R \leq 1)$. To leading order, small perturbations $\tilde{r}(x, t)$ and $\tilde{\theta}(x, t)$ of this solution satisfy

$$
\begin{aligned}
& \tilde{r}_{t}=\tilde{r}_{x x}-2 R^{2} \tilde{r}-2 R \tilde{\theta}_{x} \sqrt{1-R^{2}}, \\
& \tilde{\theta}_{t}=\tilde{\theta}_{x x}-2 \omega_{1} R \tilde{r}+\frac{2 \tilde{r}_{x} \sqrt{1-R^{2}}}{R} .
\end{aligned}
$$

Copyright $\odot$ by SIAM. Unauthorized reproduction of this article is prohibited. 
In the standard way, we look for solutions of the form $(\tilde{r}, \tilde{\theta})=(\bar{r}, \bar{\theta}) e^{\lambda t+\nu x}$, where $\bar{r}$ and $\bar{\theta}$ are constants, giving

$$
\begin{aligned}
& \lambda \bar{r}=\nu^{2} \bar{r}-2 R^{2} \bar{r}-2 \nu R \bar{\theta} \sqrt{1-R^{2}}, \\
& \lambda \bar{\theta}=\nu^{2} \bar{\theta}-2 \omega_{1} R \bar{r}+\frac{2 \nu \bar{r} \sqrt{1-R^{2}}}{R} .
\end{aligned}
$$

This implies the dispersion relation

$$
\begin{aligned}
0=\mathcal{A}(\lambda, \nu) & \equiv \lambda^{2}-2 \lambda\left(\nu^{2}-R^{2}\right)+\nu^{2}\left(\nu^{2}+4-6 R^{2}\right)-4 \omega_{1} \nu R^{2} \sqrt{1-R^{2}} \\
& =\nu^{4}+2 \nu^{2}\left(2-3 R^{2}-\lambda\right)-4 \omega_{1} \nu R^{2} \sqrt{1-R^{2}}+\lambda\left(\lambda+2 R^{2}\right)
\end{aligned}
$$

as the condition for nontrivial solutions. Note that $\mathcal{A}(0,0)=0$ for all $R$, reflecting the neutral stability of the waves to translation. Note also there is no cubic term for $\nu$ in this equation, which is a simplifying feature for the analysis below.

For stability (on the whole real line) we must consider values of $\lambda$ satisfying $\mathcal{A}(\lambda, \nu)=0$, with $\nu$ pure imaginary; the set of such $\lambda$ 's is known as the "essential spectrum," denoted by $\Sigma_{\text {ess. }}$. The condition for stability is that $\operatorname{Re}(\lambda)<0$ for all $\lambda$ in the essential spectrum, except $\lambda=0$. Kopell and Howard [22] showed that this condition reduces to $R>r_{\text {ess }}$ (defined in (3)).

To address the distinction between absolute and convective instability, we introduce the so-called absolute spectrum. To describe this, we return to the dispersion relation $\mathcal{A}(\lambda, \nu)=0$. For each $\lambda$, this has four roots for $\nu$, which we denote by $\nu_{i}(\lambda)(i=1,2,3,4)$, labeled so that

$$
\operatorname{Re}\left(\nu_{1}\right) \geq \operatorname{Re}\left(\nu_{2}\right) \geq \operatorname{Re}\left(\nu_{3}\right) \geq \operatorname{Re}\left(\nu_{4}\right)
$$

We refer to the label $i$ of $\nu_{i}(\lambda)$ in this ordering as the index of the root. The "generalized absolute spectrum" is the set of $\lambda$ such that $\operatorname{Re}\left(\nu_{i}\right)=\operatorname{Re}\left(\nu_{i+1}\right)$ for some $i=1,2$, or 3 . The "absolute spectrum" $\Sigma_{\text {abs }}$ is a subset of the generalized absolute spectrum, and for a system of two coupled reaction-diffusion equations it is the set of $\lambda$ for which $\operatorname{Re}\left(\nu_{2}\right)=\operatorname{Re}\left(\nu_{3}\right)$ (for details, see [38] and Corollary 3 below). The complement of the absolute spectrum in the generalized absolute spectrum, where the index of the repeated roots for $\nu$ in our equations is either 1 or 3 , has no direct relevance for the stability of selected wavetrains in numerical simulations or for the spectrum of the operator. We refer to the proofs in [38] for further details on this part of the generalized absolute spectrum. We calculate the full generalized absolute spectrum in this paper because it allows us to systematically compute the absolute spectrum.

Despite its name, the absolute spectrum is not a spectrum in the sense of solutions to an eigenvalue problem. However, its relevance for the spectrum on large bounded domains is as follows. Consider the spectrum $\Sigma_{L}$ of the linearization of (1) in a (near) wavetrain solution posed on the domain $(-L, L)$. Then for generic separated linear boundary conditions, $\Sigma_{\text {abs }}$ is the set of accumulation points of $\Sigma_{L}$ as $L \rightarrow \infty$. In particular, instability of the absolute spectrum implies an instability on all sufficiently large domains. Note that for periodic boundary conditions, the set of accumulation points of $\Sigma_{L}$ as $L \rightarrow \infty$ is the essential spectrum. Sandstede and Scheel [38] give a full discussion of these ideas.

Copyright ( $\odot$ by SIAM. Unauthorized reproduction of this article is prohibited. 


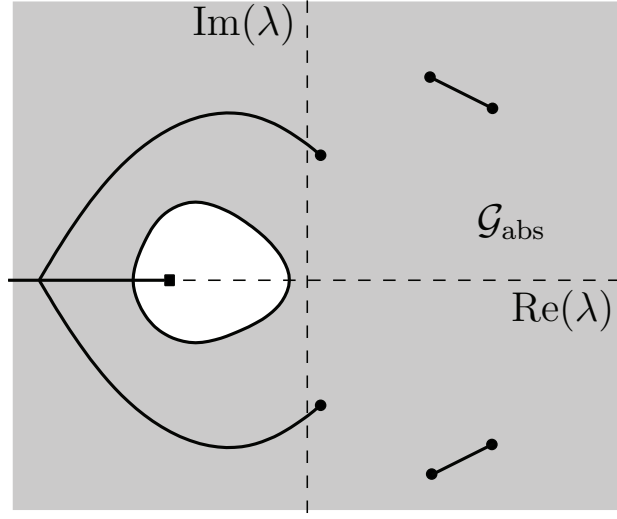

(a)

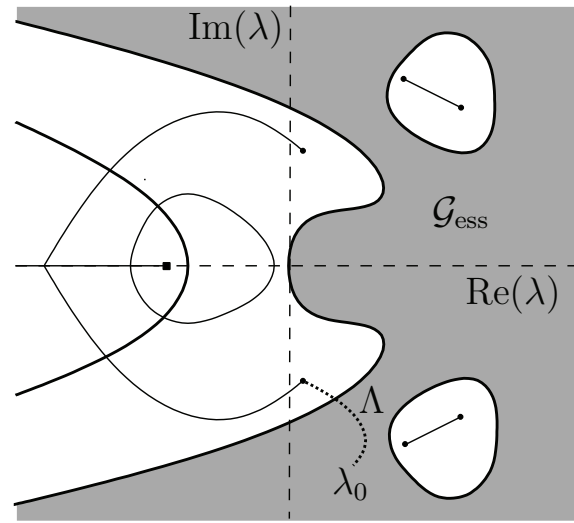

(b)

Figure 2. (a) A fictional set $\mathcal{G}_{\mathrm{abs}} \subset \mathbb{C}$ (shaded region). The solid curves are the absolute spectrum $\Sigma_{\mathrm{abs}}$, the bullets are branch points in $\partial \mathcal{G}_{\text {abs }}$, and the square a branch point in $\mathcal{G}_{\text {abs }} \backslash \partial \mathcal{G}_{\text {abs }}$. (b) A fictional set $\mathcal{G}_{\text {ess }} \subset \mathbb{C}$ (shaded region) consistent with $\mathcal{G}_{\text {abs }}$ in (a). The thick solid curves are the essential spectrum $\Sigma_{\text {ess }}$, the thin solid lines $\Sigma_{\mathrm{abs}}$, and the bullets and square are as in (a). We plot in addition a possible point $\lambda_{0}$ and curve $\Lambda$ (dotted) for the proof of Lemma 1.

The notions of absolute and convective instabilities were first developed for the whole real line [12], and in this case only simultaneous roots of $\mathcal{A}$ and $\partial_{\nu} \mathcal{A}$, referred to as branch points, are meaningful; trivially, these are contained in the generalized absolute spectrum. As mentioned, an absolute instability means that perturbations lead to pointwise growth, and this occurs when a branch point of the dispersion relation, with positive real part, satisfies the so-called pinching condition $[12,11,9,8]$, which is explained below.

We now prove a lemma that makes a connection between the pinching condition and the absolute spectrum; the result may be well known, but we are not aware of a reference. We do not attempt to explore the connection in full generality here. Rather, we focus on the lambda-omega system (1), for which the combination of the lemma and Corollary 3 below implies that absolute stability is determined by branch points in the absolute spectrum that satisfy the pinching condition. Let $\left(\lambda_{*}, \nu_{*}\right)$ be a branch point, with $\nu_{ \pm}(\lambda)$ continuous curves of solutions to $\mathcal{A}=0$ with $\nu_{ \pm}\left(\lambda_{*}\right)=\nu_{*}$. The "pinching" or "collision" condition is satisfied at $\left(\lambda_{*}, \nu_{*}\right)$ if, for suitable choices of $\nu_{ \pm}$, it holds that $\operatorname{sgn}\left(\operatorname{Re} \nu_{ \pm}(\lambda)\right)= \pm 1$ as $\operatorname{Re}(\lambda) \rightarrow+\infty$. This is satisfied if the sign condition holds for values of $\lambda$ with sufficiently large real part along any curve of $\lambda$ values emanating from $\lambda_{*}$. In fact, the real parts of all $\nu$ are unbounded, as the real part of $\lambda$ tends to infinity (see, for example, Lemma 3.3 in [32]). As defined above, we refer to solutions with unstable essential spectrum, but stable absolute spectrum, as convectively unstable; we use the term "absolutely unstable" if the absolute spectrum is unstable.

In preparation, denote by $\mathcal{G}_{\text {abs }}$ the connected component of $\mathbb{C} \backslash \Sigma_{\text {abs }}$ that contains an unbounded part of the real axis, and by $\mathcal{G}_{\text {ess }}$ the connected component of $\mathbb{C} \backslash \Sigma_{\text {ess }}$ that contains an unbounded part of the real axis (illustrated schematically in Figure 2); these sets are well defined [38, 39].

Lemma 1.

1. All branch points $\left(\lambda_{*}, \nu_{*}\right)$ for which $\lambda_{*}$ lies on the boundary $\partial \mathcal{G}_{\text {abs }}$ satisfy the pinching 
condition.

2. At all branch points $\left(\lambda_{*}, \nu_{*}\right)$ satisfying the pinching condition, $\lambda_{*}$ lies in $\mathbb{C} \backslash \mathcal{G}_{\text {abs }}$, so that $\operatorname{Re}\left(\lambda_{*}\right) \leq \max \operatorname{Re} \Sigma_{\text {abs }}$.

Proof. It is well known that for a system of two coupled reaction-diffusion equations such as (1) the Morse index $i(\lambda):=\#\{\nu(\lambda): \operatorname{Re}(\nu(\lambda)) \geq 0\}$ of any $\lambda \in \mathcal{G}_{\text {ess }}$ is $i(\lambda)=2$, and that $\mathcal{G}_{\text {ess }} \cap \Sigma_{\text {abs }}=\emptyset$ (see, for example, [37]). The idea underlying our proof is to look for changes in the ordering of the real parts of $\nu(\lambda)$ along a curve $\Lambda$ in the complex $\lambda$ plane connecting the branch point $\lambda_{*}$ to a point $\lambda_{0} \in \mathcal{G}_{\text {ess }}$; see Figure 2(b) for an illustration. From the definition of $\mathcal{G}_{\text {ess }}$, the Morse index $i\left(\lambda_{0}\right)$ is the same for all $\lambda_{0}$ in this region. In the following we label curves with indices denoting the ordering at $\lambda_{*}$ : we denote by $\tilde{\nu}_{j}(\lambda)$ the continuation of $\nu_{j}\left(\lambda_{*}\right)$ along $\Lambda$; i.e., $\tilde{\nu}_{j}$ is continuous and $\tilde{\nu}_{j}\left(\lambda_{*}\right)=\nu_{j}\left(\lambda_{*}\right)$. Note that since $\lambda_{*} \in \Sigma_{\text {abs }}$ and is a branch point, $\nu_{2}\left(\lambda_{*}\right)=\nu_{3}\left(\lambda_{*}\right)$.

Item 1 . Since $\lambda_{*} \in \partial \mathcal{G}_{\text {abs }}$ we can choose $\Lambda$ so that $\Lambda \cap \Sigma_{\text {abs }}=\left\{\lambda_{*}\right\}$. The pinching condition fails if and only if $\operatorname{sgn}\left(\operatorname{Re}\left(\tilde{\nu}_{2}\left(\lambda_{0}\right)\right)\right)=\operatorname{sgn}\left(\operatorname{Re}\left(\tilde{\nu}_{3}\left(\lambda_{0}\right)\right)\right)$, and we suppose this to be the case. Since $i\left(\lambda_{0}\right)=2$ it follows that either $\operatorname{Re}\left(\tilde{\nu}_{1}(\lambda)\right)$ or $\operatorname{Re}\left(\tilde{\nu}_{4}(\lambda)\right)$ has to change sign along $\Lambda$, and we next show that this implies the contradiction $\Lambda \cap \Sigma_{\text {abs }} \neq\left\{\lambda_{*}\right\}$.

Suppose that $\operatorname{sgn}\left(\operatorname{Re}\left(\tilde{\nu}_{2,3}\left(\lambda_{0}\right)\right)\right)=\operatorname{sgn}\left(\operatorname{Re}\left(\nu_{*}\right)\right)=-1$. Since $i\left(\lambda_{0}\right)=2$ and $\nu_{*}=\nu_{2}\left(\lambda_{*}\right)$, it follows that $\operatorname{Re}\left(\tilde{\nu}_{1}\left(\lambda_{0}\right)\right)>0$. Therefore the $\nu_{j}$ 's reorder along $\Lambda$ in a way that gives $\tilde{\nu}_{1}\left(\lambda_{0}\right)=$ $\nu_{j}\left(\lambda_{0}\right)$ for $j=2$ or $j=3$. Hence there are points $\lambda_{\ell} \in \Lambda(\ell=2,3)$ different from $\lambda_{*}$ at which $\operatorname{Re}\left(\tilde{\nu}_{1}\left(\lambda_{\ell}\right)\right)=\operatorname{Re}\left(\nu_{\ell}\right)$. Since the ordering changes in increments of one (or simultaneously), one of these points lies in $\Sigma_{\text {abs }}$, which contradicts $\Lambda \cap \Sigma_{\text {abs }}=\left\{\lambda_{*}\right\}$. Hence, $\operatorname{sgn}\left(\operatorname{Re}\left(\tilde{\nu}_{2,3}\left(\lambda_{0}\right)\right)\right)=$ $\operatorname{sgn}\left(\operatorname{Re}\left(\nu_{*}\right)\right)=-1$ cannot hold true.

The same argument, now applied to $\tilde{\nu}_{3}(\lambda)$, shows that $\operatorname{sgn}\left(\operatorname{Re}\left(\tilde{\nu}_{2,3}\left(\lambda_{0}\right)\right)\right)=\operatorname{sgn}\left(\operatorname{Re}\left(\nu_{*}\right)\right)=$ +1 also contradicts the assumption. In the cases $\operatorname{sgn}\left(\operatorname{Re}\left(\nu_{2,3}\left(\lambda_{0}\right)\right)\right)=-\operatorname{sgn}\left(\operatorname{Re}\left(\nu_{*}\right)\right)$ the same argument applies again, with $\tilde{\nu}_{1}(\lambda)$ if $\operatorname{sgn}\left(\operatorname{Re}\left(\nu_{*}\right)\right)=+1$ and $\tilde{\nu}_{3}(\lambda)$ otherwise.

In conclusion, $\operatorname{Re}\left(\tilde{\nu}_{2,3}\left(\lambda_{0}\right)\right)$ must have opposite signs, which is the pinching condition. Note that $\lambda_{0}$ can be chosen so that $\operatorname{Re}\left(\tilde{\nu}_{2,3}\left(\lambda_{0}\right)\right) \neq 0$.

Item 2. Note that $\lambda_{*} \notin \mathcal{G}_{\text {ess }}$ since all points in $\mathcal{G}_{\text {ess }}$ have the same Morse index, whereas the pinching condition requires a change. We choose a curve $\Lambda$ connecting $\lambda_{*}$ to an arbitrary point $\lambda_{0} \in \mathcal{G}_{\text {ess }}$ and will show that $\Lambda \cap \Sigma_{\text {abs }} \neq \emptyset$.

We assume the nontrivial case $\lambda_{*} \notin \Sigma_{\text {abs }}$ so that $\nu_{*}=\nu_{\ell}\left(\lambda_{*}\right)=\nu_{\ell+1}\left(\lambda_{*}\right)$ for $\ell=1$ or $\ell=3$. The pinching condition implies $\operatorname{Re}\left(\tilde{\nu}_{\ell}\left(\lambda_{0}\right)\right)<0<\operatorname{Re}\left(\tilde{\nu}_{\ell+1}\left(\lambda_{0}\right)\right)$ so that either $\operatorname{Re}\left(\tilde{\nu}_{\ell}(\lambda)\right)$ or $\operatorname{Re}\left(\tilde{\nu}_{\ell+1}(\lambda)\right)$ changes sign along $\Lambda$. Since $i\left(\lambda_{0}\right)=2$ another sign change must also occur; i.e., $\operatorname{sgn}\left(\operatorname{Re}\left(\tilde{\nu}_{k}\left(\lambda_{*}\right)\right)\right) \neq \operatorname{sgn}\left(\operatorname{Re}\left(\tilde{\nu}_{k}\left(\lambda_{0}\right)\right)\right)$ for either $k \equiv \ell+2 \bmod 4$ or $k \equiv \ell+3 \bmod 4$. The real parts of the sign-changing solutions must be the same at least once along $\Lambda$. As in item 1 , if there is one crossing, then there is (also) one in $\Sigma_{\text {abs }}$.

3.1. The most unstable points of the absolute spectrum. The dynamics after the onset of instability of the absolute spectrum depend on the way in which it becomes unstable. Concerning the distinction between the absolute spectrum and the pinching condition, the question is whether the absolute spectrum becomes unstable via a branch point. If not, one has a so-called remnant instability. In such a case, all linear modes decay in a stationary frame of reference, but there are unstable modes that grow while traveling to either the left or the right, enabling a perturbation to grow at its original site via repeated reflections off 
the boundaries. We are aware of only one example in which this has been demonstrated numerically (see Figure 10 of [33]). On the other hand, there do not seem to be any rigorous results showing that the onset of absolute instability occurs via branch points, except in trivial cases. The main difficulty lies in the algebraic complications that arise even for rather simple dispersion relations. In the following we overcome these difficulties for a certain class of dispersion relations and deduce that the most unstable points in the absolute spectrum for $\mathcal{A}$ are branch points. Specifically, we consider a generalization of $\mathcal{A}$ given by $d: \mathbb{C}^{2} \rightarrow \mathbb{C}$ of the form

$$
d(\lambda, \nu)=\lambda^{2}-2 \lambda\left(\nu^{2}-A\right)+\nu^{4}+B \nu^{2}+C \nu
$$

with constants $A, B, C \in \mathbb{R}$. The roots $\lambda(\nu)$ of $d(\lambda, \nu)=0$ come in the two functions

$$
\lambda_{ \pm}=\nu^{2}-A \pm \sqrt{-\nu^{2}(2 A+B)-C \nu+A^{2}} .
$$

Theorem 2. If the absolute spectrum $\Sigma_{\text {abs }}$ of $d(\lambda, \nu)$ as in (10) with $2 A+B>0$ contains a branch point $\left(\lambda_{*}, \nu_{*}\right)$, where $\operatorname{Im}\left(\lambda_{*}\right) \neq 0$, then the following hold:

1. $\lambda_{*}$ is one of the two unique complex conjugate points that have maximal real part within $\Sigma_{\text {abs }}$.

2. $\lambda_{*} \in \partial \mathcal{G}_{\mathrm{abs}}$, and $\left(\lambda_{*}, \nu_{*}\right)$ satisfies the pinching condition.

3. If additionally $C \neq 0$, then a branch point at the origin cannot lie in the absolute spectrum but instead has to be in the generalized absolute spectrum with index 1 or 3 .

Before proving this result, we point out its significance for our purposes.

Corollary 3. If the absolute spectrum $\Sigma_{\mathrm{abs}}$ of a wavetrain (2) of the lambda-omega system (1) for $0<R<1$ and $\omega_{1}>0$ contains a branch point $\left(\lambda_{*}, \nu_{*}\right)$ with $\operatorname{Im}\left(\lambda_{*}\right) \neq 0$, then $\lambda_{*}$ is one of the two unique complex conjugate points that have maximal real part within $\Sigma_{\text {abs }}$. Such a branch point lies in $\partial \mathcal{G}_{\mathrm{abs}}$ and satisfies the pinching condition. Moreover, a branch point at the origin cannot lie in the absolute spectrum.

Proof. For the dispersion relation of wavetrains in the lambda-omega system (1) we have $A=R^{2}>0, B=4-6 R^{2}$ so that $2 A+B=4\left(1-R^{2}\right)>0$, and $C=-4 \omega_{1} R^{2} \sqrt{1-R^{2}}<0$.

The proof of Theorem 2 is based on the following observation.

Lemma 4. For any fixed $\eta \in \mathbb{R}$ the real parts of $\lambda_{ \pm}(\eta+\mathrm{i} k)$ are even functions of $k$, and the imaginary parts are odd functions. If $2 A+B>0$, then the following hold:

1. $\operatorname{Re}\left(\lambda_{-}(\eta+\mathrm{i} k)\right)+\operatorname{Re}\left(\lambda_{+}(\eta+\mathrm{i} k)\right)$ and $\operatorname{Re}\left(\lambda_{-}(\eta+\mathrm{i} k)\right)-\operatorname{Re}\left(\lambda_{+}(\eta+\mathrm{i} k)\right)$ are both strictly decreasing in $k \geq 0$.

2. For $k>0$ it holds that $\operatorname{Re}\left(\lambda_{-}(\eta+\mathrm{i} k)\right)$ is strictly decreasing in $k$, and that $\operatorname{Re}\left(\lambda_{+}(\eta+\mathrm{i} k)\right)$ either is strictly decreasing or is strictly increasing on $0<k<k_{*}$ and strictly decreasing on $k>k_{*}$, for some $k_{*}>0$.

Proof. Since the coefficients of $d$ are real, the real and imaginary parts are respectively even and odd functions of $k$. To facilitate a more detailed study, we write $D=2 A+B$, $z=-\nu^{2} D-C \nu+A^{2}$, and $\nu=\eta+\mathrm{i} k$, giving

$$
L_{ \pm}(k):=\operatorname{Re}\left(\lambda_{ \pm}(\eta+\mathrm{i} k)\right)=\eta^{2}-k^{2}-A \pm \sqrt{\frac{|z|+\operatorname{Re}(z)}{2}} .
$$

Copyright (c) by SIAM. Unauthorized reproduction of this article is prohibited. 
We readily compute

$$
\begin{aligned}
& \operatorname{Re}(z)=D k^{2}-D \eta^{2}-\eta C+A^{2}, \\
& \operatorname{Im}(z)=-(2 \eta D+C) k,
\end{aligned}
$$

and with $F=2 \eta^{2} D^{2}+2 \eta D C+2 D A^{2}+C^{2}, G=-D \eta^{2}-\eta C+A^{2}$, we obtain

$$
|z|^{2}=\operatorname{Re}(z)^{2}+\operatorname{Im}(z)^{2}=D^{2} k^{4}+F k^{2}+G^{2} .
$$

Item 1. $F=2(\eta D+C / 2)^{2}+2 D A^{2}+C^{2} / 2 \geq 0$. Hence, for all $\eta$ we have that $|z|^{2}$ is an even function of $k$ and monotone increasing in $k \geq 0$, so that $|z|$ has the same properties. Since $\operatorname{Re}(z)$ also has these properties, so does $|z|+\operatorname{Re}(z) \geq 0$. Finally, since $|z|+\operatorname{Re}(z) \geq 0$, $\sqrt{|z|+\operatorname{Re}(z)}$ also has these properties.

Item 2. It follows that $\operatorname{Re}\left(\lambda_{-}(\eta+\mathrm{i} k)\right)$ is the sum of two even functions of $k$ that are monotone decreasing in $k \geq 0$, and therefore it has these properties itself. However, $\operatorname{Re}\left(\lambda_{+}(\eta+\mathrm{i} k)\right)$ is the sum of an increasing and a decreasing function, and therefore it requires more careful investigation. Writing $K=k^{2}$,

$$
\frac{\partial}{\partial k} L_{+}(k)=2 k\left(-1+\frac{1}{2 \sqrt{2}} \frac{\partial|z| / \partial K+\partial \operatorname{Re}(z) / \partial K}{\sqrt{|z|+\operatorname{Re}(z)}}\right) .
$$

Differentiating again shows that if $\partial L_{+}(k) / \partial k=0$ at $k=k_{*}>0$, then

$$
\left.\frac{\partial^{2}}{\partial k^{2}} L_{+}(k)\right|_{k=k_{*}}=\frac{k}{\sqrt{2(|z|+\operatorname{Re}(z))}}\left[\frac{\partial^{2}|z|}{\partial K^{2}}-4\right],
$$

and differentiation of (11) shows that

$$
\frac{\partial^{2}|z|}{\partial K^{2}}=\frac{4 D^{2} G^{2}-F^{2}}{4\left(D^{4} K^{2}+F K+G^{2}\right)^{3 / 2}} .
$$

Moreover, $4 D^{2} G^{2}-F^{2}=-(2 D \eta+C)^{2}\left(4 D A^{2}+C^{2}\right) \leq 0$. Therefore at any point $k_{*}>0$ at which $\partial L_{+} / \partial k=0, \partial^{2} L_{+} / \partial k^{2}$ is strictly negative. Finally, we note that $L_{+} \rightarrow-\infty$ as $k \rightarrow \infty$. Therefore $L_{+}(k)$ must have one of the two forms given in item 2 .

Proof of Theorem 2. In the following we use the notation of the proof of Lemma 4.

Item 1 . We begin by applying Lemma 4 with $\eta=\operatorname{Re}\left(\nu_{*}\right)$; due to complex conjugation symmetry, we can assume without loss of generality that $k \geq 0$. Since the branch point is a double root of $d$ with respect to $\nu$, either $\lambda_{*}=\lambda_{+}\left(\nu_{*}\right)$ and $\partial \lambda_{+} / \partial \nu\left(\nu_{*}\right)=0\left(\Rightarrow \partial \lambda_{+} / \partial k\left(\nu_{*}\right)=0\right)$ or $\lambda_{*}=\lambda_{-}\left(\nu_{*}\right)$ and $\partial \lambda_{-} / \partial \nu\left(\nu_{*}\right)=0\left(\Rightarrow \partial \lambda_{-} / \partial k\left(\nu_{*}\right)=0\right)$. Referring to item 2 of Lemma 4 , the only case that is consistent with this is $\lambda_{*}=\lambda_{+}\left(\nu_{*}\right)$, with $\lambda_{*}$ being nonmonotonic in $k$, and with $\operatorname{Im}\left(\nu_{*}\right)=k_{*}$. Since $L_{-}-L_{+}$is negative at $k=0$ and decays monotonically for $k \geq 0$ (item 1 of Lemma 4), we have that $\operatorname{Re}\left(\lambda_{*}\right)>\max \left\{L_{-}(k): k \in \mathbb{R}\right\}$.

In conclusion, the branch point lies at the maximal real part of the roots of $d(\lambda, \eta+i k)$ $(k \in \mathbb{R})$. We now note that these roots define the essential spectrum in an exponentially weighted space with weight $\eta$. Lemma 3.3, Lemma 4.1, and Remark 1 of [32] together show that for a wavetrain solution of any reaction-diffusion system in one space dimension, the most 
unstable point of a weighted essential spectrum is at least as unstable as the most unstable point of the absolute spectrum. Therefore in the present case, the weighted essential spectrum and the absolute spectrum must coincide at $\left(\lambda_{*}, \nu_{*}\right)$, which is the most unstable point of each spectrum.

Item 2. $\lambda_{*} \in \partial \mathcal{G}_{\text {abs }}$ follows immediately from the proof of item 1 . Lemma 1 then implies that the branch point satisfies the pinching condition.

Item 3 . If the origin is a branch point, then

$$
d(0, \nu)=\nu\left(\nu^{3}+B \nu+C\right)=0,
$$

whose solutions are $\nu=0$ and the three roots of a cubic polynomial. Since this cubic does not have a quadratic term, the sum of its roots vanishes. Therefore if $C \neq 0$, so that none of these three roots is zero, the roots must be $\nu_{0}, \nu_{0}$, and $-2 \nu_{0}$ for some $\nu_{0} \neq 0$. Therefore a branch point must have index equal to either $1\left(\nu_{0}>0\right)$ or $3\left(\nu_{0}<0\right)$ : its index cannot be 2 , and thus it cannot be part of the absolute spectrum.

\section{Numerical calculation of the absolute spectrum.}

4.1. Numerical methodology. Rademacher, Sandstede, and Scheel [33] give a detailed account of the use of numerical continuation to calculate the absolute spectrum; here we give a summary for the specific case of the lambda-omega equations (1), with further details provided in the supplementary online material [49]. Alternative approaches to the numerical calculation of absolute stability (on the real line) are given in [11, 10, 7, 54, 52, 51, 53]. For any given $\lambda$, (8) can be written in matrix form as

$$
\begin{gathered}
\mathcal{D}\left(\lambda, \nu_{k}\right) \mathbf{U}_{k}=0, \\
\text { where } \mathcal{D}(\lambda, \nu)=\mathbf{I} \mathbf{d} \nu^{2}+\mathbf{C} \nu+\mathbf{F}-\lambda, \quad \mathbf{U}_{k}=\left(\begin{array}{c}
\bar{r}_{k} \\
\bar{\theta}_{k}
\end{array}\right), \\
\mathbf{C}=\left(\begin{array}{cc}
0 & -2 R \sqrt{1-R^{2}} \\
2 \sqrt{1-R^{2}} / R & 0
\end{array}\right), \quad \mathbf{F}=\left(\begin{array}{cc}
-2 R^{2} & 0 \\
-2 \omega_{1} R & 0
\end{array}\right),
\end{gathered}
$$

and $\mathbf{I d}$ is the $2 \times 2$ identity matrix. Here $\nu_{1}, \ldots, \nu_{4}$ are the four roots of the dispersion relation (9). Taking real and imaginary parts of (13) gives 16 equations to be solved simultaneously. Following [33], we perform numerical continuations of solutions of these equations using the software package AUTO $[16,15,17]$. Specifically, we continue in $\lambda$ from known solution values and monitor the resulting changes in $\nu_{k}$. We ensure that the continuations remain on the generalized absolute spectrum by adding the constraint that two of the values of $\nu_{k}$ must have the same real part. Thus, instead of representing the real parts of the two $\nu$ values separately, we use a single AUTO parameter for their common real part.

Starting solutions are necessary to begin the continuations. To obtain these we utilize Corollary 3 and the general finding of [33] that for constant coefficient problems all curves of the generalized absolute spectrum emanate from branch points, with the absolute spectrum being a connected subset.

Concerning branch points of $\mathcal{A}$, note that $\partial_{\nu} \mathcal{A}=0$ means that

$$
\lambda=\nu^{2}+2-3 R^{2}-\frac{\omega_{1} R^{2} \sqrt{1-R^{2}}}{\nu} .
$$

Copyright $\odot$ by SIAM. Unauthorized reproduction of this article is prohibited. 
To calculate the position of the branch points, we first substitute (14) into the dispersion relation (9). This gives a fourth order polynomial in $\nu$ (which we omit for brevity), zeros of which give four $\nu$ values that are double roots of $\mathcal{A}$.

We then calculate the four $\lambda$ values associated with these double roots by substituting the $\nu$ values back into (14). (In the supplementary online material [49] we give MATLAB [25] code that calculates these values numerically.) We can also substitute the $\lambda$ values associated with each branch point into (9) and solve for $\nu$ to give the other two $\nu$ values at any given branch point. This allows us to classify the nature of the branch points in terms of the index of the repeated root for $\nu$.

This procedure reveals that for wavetrain solutions of the lambda-omega equations (1), the branch points are either four real $\lambda$ values or two real $\lambda$ 's plus a complex conjugate pair. Note that Theorem 2 excludes the possibility of two complex conjugate pairs of branch points. Figure 3 shows these $\lambda$ 's for the wavetrain selected by the zero Dirichlet boundary condition, as a function of $\omega_{1}$. As $\omega_{1}$ is increased from zero there are initially four real $\lambda$ values at the branch points, and at $\omega_{1} \approx 0.8$ two of these become a complex conjugate pair.

Using branch points as starting solutions for the continuations raises a technical difficulty: AUTO cannot distinguish the two equal values of $\nu_{k}$ when performing the first continuation step from the branch point. Rademacher, Sandstede, and Scheel [33] developed a "desingularization" procedure to overcome this complication. For a branch point at which $\nu_{j}=\nu_{j+1}$, this involves replacing (13) for $k=j+1$ with

$$
\left[\mathbf{I d}\left(2 \nu_{j}+\mathrm{i} \eta\right)+\mathbf{C}\right]\left(\mathbf{U}_{j}+\mathrm{i} \eta \mathbf{V}\right)+\mathcal{D}\left(\lambda, \nu_{j}\right) \mathbf{V}=0
$$

where $\mathbf{V}$ is an appropriate vector such that (15) is satisfied, i is the imaginary unit, and initially $\eta=0$. Continuation can then proceed by starting at the branch point and solving (13) for the three $\nu_{k}$ with $k \neq j+1$ (12 equations) together with (15) (4 equations). We obtain nontrivial initial values for $\mathbf{U}_{k}(k \neq j+1)$ by substituting the appropriate values of $\nu_{k}, \lambda, \omega_{1}, R$ into (8a) to give an equation of the form $a_{k} \bar{r}_{k}+b_{k} \bar{\theta}_{k}=0$; we take $\bar{r}_{k}=-b_{k} / \sqrt{a_{k}^{2}+b_{k}^{2}}$ and $\bar{\theta}_{k}=a_{k} / \sqrt{a_{k}^{2}+b_{k}^{2}}$. We do not initially define nontrivial solutions for $\mathbf{V}$; rather, we choose this vector arbitrarily and leave AUTO to find appropriate values in the initial continuation steps. Continuation in $\lambda$ from initial values at the branch points therefore involves three stages. The first stage is to perform a continuation in a dummy parameter for a low number of iterations, while tracking all the other solution parameters, to identify appropriate values for $\mathbf{V}$. The second stage involves a continuation in $\eta$, from $\eta=0$, using the desingularized equations (15), to obtain the four separate values of $\nu_{k}$, two of which will have equal real parts. This continuation stage can be run for as long as is desired or possible; however, it is generally more convenient to switch to (13) for longer continuations and continue in $\operatorname{Re}(\lambda)$. In the supplementary online material [49] we give more details of all these continuation stages, plus example code and output; we refer the reader to those for more details.

Example output from this procedure is shown in Figure 4. For the case $\omega_{1}=1$ used in Figure 4 (corresponding to the simulations shown in Figure 1(a),(b)), two of the four branch points are on the real line. Continuations starting from the branch point at $\operatorname{Re}(\lambda) \approx 6$ towards $\operatorname{Re}(\lambda)=+\infty$ show two pairs of $\nu_{k}$ values with equal real parts (index $=1$ ). This is also true for continuations from the branch point at $\operatorname{Re}(\lambda) \approx-2$ towards $\operatorname{Re}(\lambda)=-\infty$. The other two

Copyright (c) by SIAM. Unauthorized reproduction of this article is prohibited. 

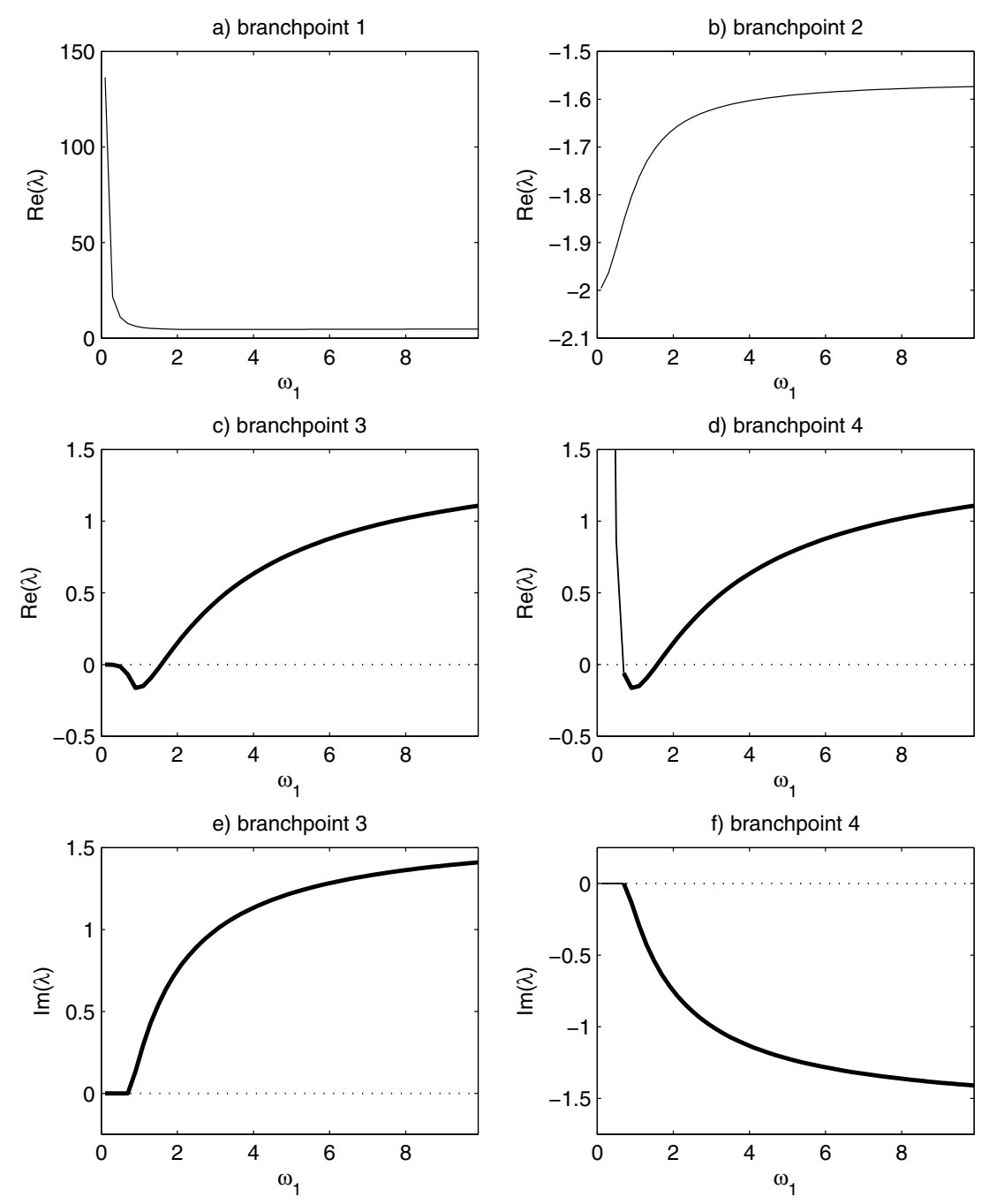

Figure 3. The relationship between $\omega_{1}$ and the real and imaginary parts of $\lambda$ at the four branch points associated with the wave selected by zero Dirichlet boundary conditions in (1). Bold lines denote $\lambda$ values for which the condition $\operatorname{Re}\left(\nu_{2}\right)=\operatorname{Re}\left(\nu_{3}\right)$ is satisfied, indicating that they are part of the absolute spectrum. At two of the four branch points, the values of $\lambda$ are real for all values of $\omega_{1}$ shown here. At the other two branch points, $\lambda$ is real for $\omega_{1}$ less than about 0.8 and complex otherwise.

branch points (again for $\omega_{1}=1$ ) are complex conjugate pairs in $\lambda$ (with $\operatorname{Re}(\lambda) \approx-0.1$ ), and a continuation towards $\operatorname{Re}(\lambda)=-\infty$ starting from one of these branch points is shown in Figure 4(b). The four $\nu_{k}$ values for this continuation split into a pair with equal $\operatorname{Re}(\nu)$ and two others with different $\operatorname{Re}(\nu)$. During the course of the continuation from the branch point, the $\operatorname{Re}\left(\nu_{k}\right)$ values change relative order such that the pair with equal real part has index 2 initially, and this changes to index 3 before changing back again to index 2. As mentioned, for a constant coefficient case such as this, the absolute spectrum (index 2) is connected [33];

Copyright $\odot$ by SIAM. Unauthorized reproduction of this article is prohibited. 

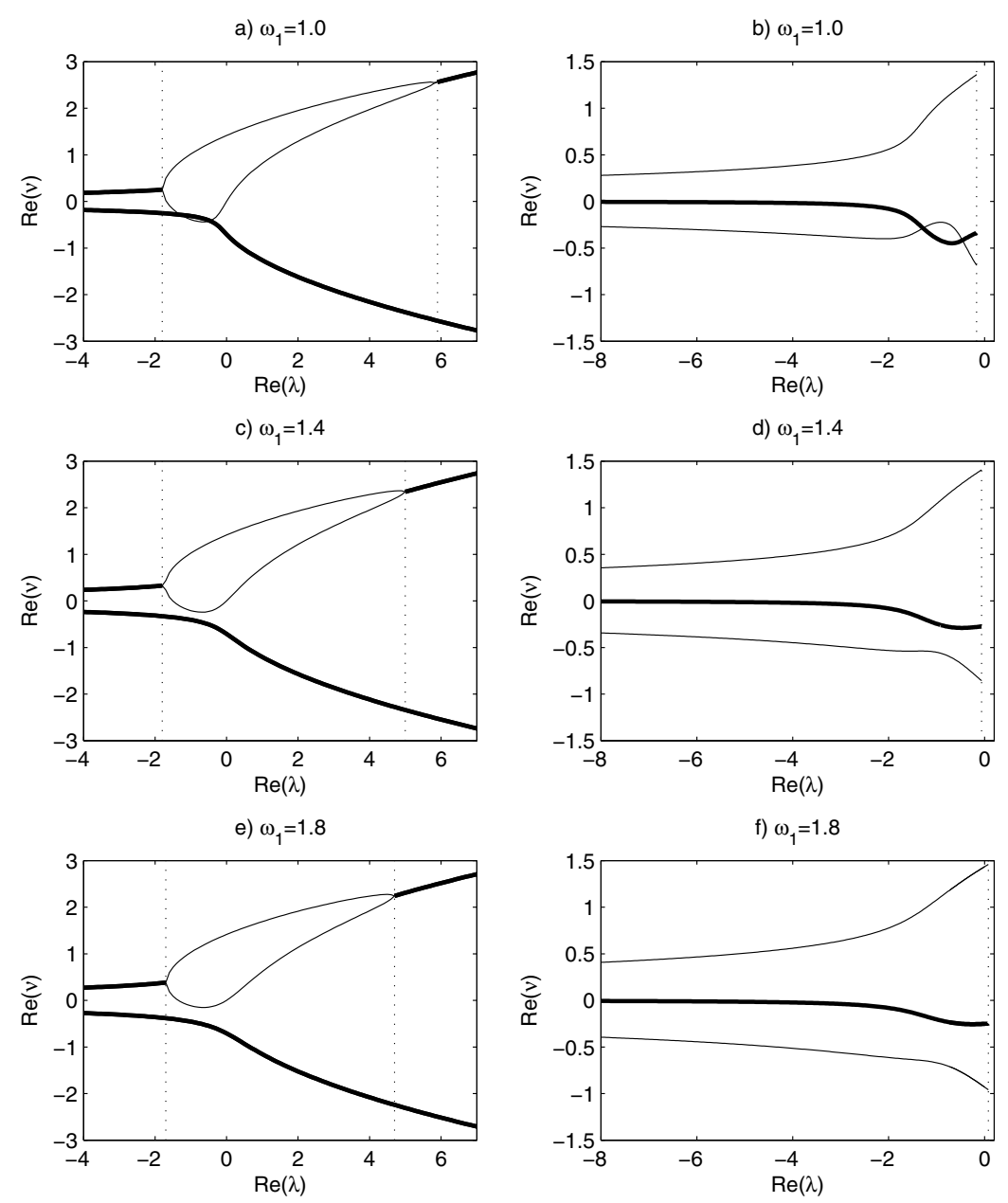

Figure 4. Example continuation results for three values of $\omega_{1}$. In each graph we plot the real parts of the four $\nu$ values for varying $\lambda$. Thick lines indicate two $\nu$ values with the same real part, and thin lines are $\nu$ values whose real part is not repeated. The vertical dashed lines indicate the branch points at which continuations were started; "branch points" are the $\lambda$ values at which there is a repeated root for $\nu$, and are given by (9) and (14). Parts (a), (c), and (e) show continuations starting from either of the real $\lambda$ values at the branch points ( $\lambda$ is real throughout these continuations), while (b), (d), and (f) show one continuation starting from one of the complex $\lambda$ branch points (the imaginary part of $\lambda$ varies in these continuations).

therefore there must be another part of the spectrum with index 2 associated with the $\operatorname{Re}(\lambda)$ values in Figure 4(b) that have index 3.

The term "triple point" denotes a point at which three of the values of $\operatorname{Re}\left(\nu_{k}\right)$ are equal; thus the points at which the lines in Figures 4(a) and 4(b) cross are triple points. Rademacher, Sandstede, and Scheel [33] show that such points are the location of bifurcations of the generalized absolute spectrum. In the region between the triple points shown in both Figures 4(a) and 4(b), there are three lines of generalized absolute spectrum in the complex $\lambda$ plane, one with index 2 for the pair of $\nu_{k}$ values with equal real part, which lies on the real line 


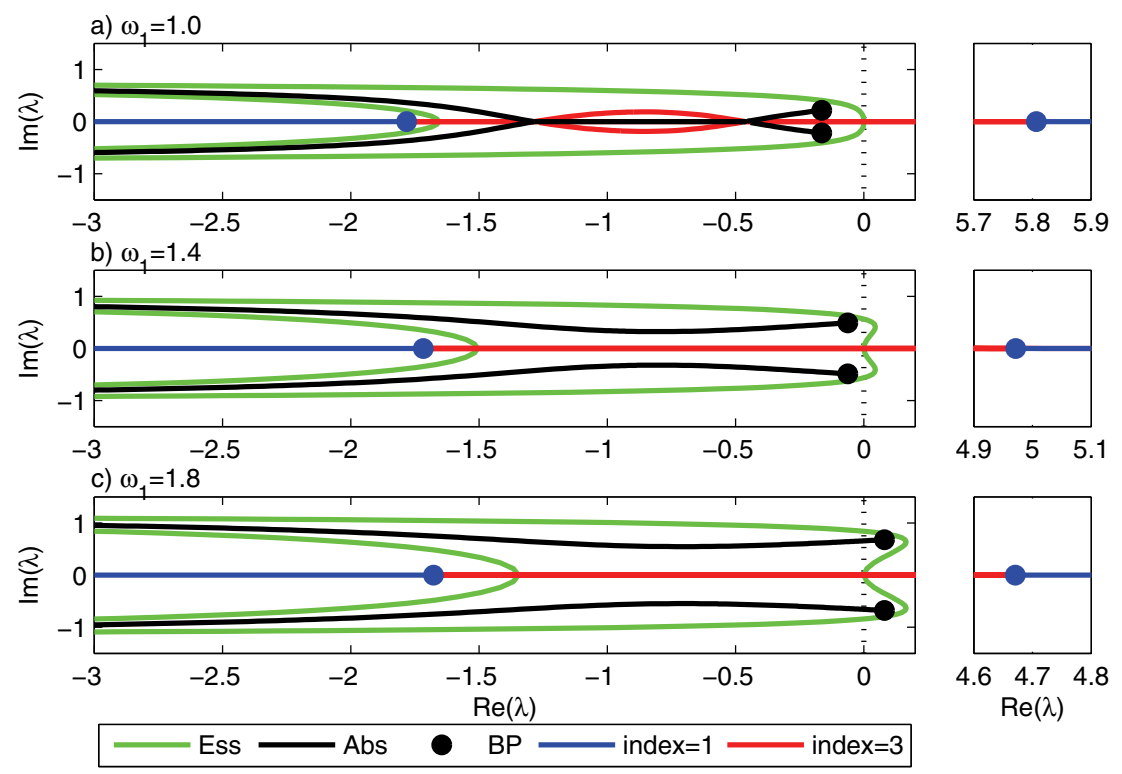

Figure 5. Essential and absolute spectra for wavetrain solutions selected by zero Dirichlet boundary conditions, with amplitudes given by (4), for different values of $\omega_{1} . \omega_{1}=1.0$ generates stable waves, $\omega_{1}=1.4$ generates essentially unstable but absolutely stable waves, and $\omega_{1}=1.8$ generates absolutely unstable waves. The numerical simulations corresponding to these solutions are shown in Figure 1. The essential spectrum (green line) was calculated using (9), assuming $\nu=i \gamma(\gamma \in \mathbb{R})$. The absolute spectrum (black line) and generalized absolute spectra (red and blue lines) were calculated using the techniques described in section 4 . The colors indicate the index of the values of $\nu$ with equal real parts (blue $=1$, black $=2$, red $=3$ ). We also plot the locations of all four branch points (dots), which we use as starting points for the continuations. For clarity we use a separate panel on the right of each of the larger panels to indicate the location of the branch point with the largest $\operatorname{Re}(\lambda)$. This branch point is not in the absolute spectrum and thus has no relevance for determining the stability of the wavetrain solutions.

(Figure 4(a)), and two others with index 3, of which one is shown in Figure 4(b). Technical details of how we switched continuation branches at triple points are given in the supplementary online material [49]. Figures 4(c)-(f) show similar continuations, starting at the branch points, for two other values of $\omega_{1}$.

4.2. Essential and absolute spectra of selected wavetrains. The data in Figure 4, in addition to data from the continuations starting at the complex conjugate branch points not shown in Figure 4, were used to construct the generalized absolute spectra and absolute spectra shown in Figure 5. We have also added the essential spectrum to these figures. Figure 5 shows that for all three values of $\omega_{1}$ there are four branch points, two with real $\lambda$ and two whose $\lambda$ values are a complex conjugate pair. For $\omega_{1}=1.0$ both the absolute spectrum (black lines in Figure 5) and the essential spectrum (green lines in Figure 5) are fully contained in the left-hand complex plane, indicating that the wavetrain is stable. For $\omega_{1}=1.4$ the essential spectrum extends into the right-hand complex plane, but the absolute spectrum does not, indicating that the wavetrain solution is convectively unstable. Comparison of Figures 5(a) and 5(b) illustrates that the onset of instability (on the whole real line) occurs through an "Eckhaus" or "sideband" instability. For $\omega_{1}=1.8$ both spectra extend into the right-hand 
half of the complex plane, indicating that the wavetrain is absolutely unstable and that the onset of absolute instability (again on the whole real line) occurs through a complex conjugate pair of branch points, as predicted by Corollary 3. We remark that this suggests that on large domains with separated boundary conditions the onset of instability is of Hopf type, provided that the boundary conditions give stable "boundary spectrum" [38].

The results shown in Figure 5 are typical for all combinations of $\omega_{1}$ and $R$ that we have looked at, except that all four $\lambda$ values associated with the four different branch points are real in some cases (see Figure 3). Trivially, the absolute spectrum has a maximum real part for $\lambda$ and extends to infinity in the left-hand half of the complex plane. Moreover, as mentioned above, in a constant coefficient case such as this, the absolute spectrum is connected, and "isolas" cannot exist. It is possible, in general, that part of the absolute spectrum that is connected to a branch point with $\operatorname{Re}(\lambda)<0$ nevertheless crosses into the right-hand half of the complex plane before curving back across the imaginary axis, leading to a "remnant" instability on large bounded domains with separated boundary conditions (see [38] for details and Figure 10 of [33] for an example). Corollary 3 shows that this cannot occur for (1).

Crucially, the presence of the absolute spectrum in the right-hand half of the complex plane is always associated with a branch point with $\operatorname{Re}(\lambda)>0$. Hence as parameter values are varied so that the selected wavetrain changes its absolute stability, this occurs via a branch point crossing the imaginary axis in $\lambda$. One simple consequence of this is that numerical calculation of the full absolute spectrum is not necessary to determine whether or not a given wavetrain is absolutely stable. Instead, one can simply calculate the positions of the four branch points in the complex $\lambda$ plane, and the associated values of $\nu$.

4.3. Thresholds for absolute stability. We calculated the wavetrain amplitude, $R$, associated with a complex conjugate pair of branch points lying on the imaginary axis, for varying $\omega_{1}$, using continuation in AUTO. This method gives the general wavetrain amplitude threshold $(R)$ for the absolute stability of wavetrain solutions to (1) as a function of $\omega_{1}$. To do this we implemented the numerical methodology described in section 4.1. We used a solution to equations (13) from a known complex conjugate branch point as a starting point, and performed a continuation in $R$ (with $\omega_{1}$ constant), labeling the solution at which $\operatorname{Re}(\lambda)=0$. We then performed a second continuation from this labeled solution, fixing $\operatorname{Re}(\lambda)=0$ and varying $\omega_{1}$ while tracking $R$. The resulting absolute stability line is plotted in Figure 6 (the thick solid line). As $\omega_{1}$ is increased from zero, the critical amplitude at which absolute stability changes declines initially before then increasing monotonically. This contrasts with the convective stability threshold (derived from (3)), which increases monotonically as $\omega_{1}$ increases from zero (the dotted line in Figure 6).

The two general stability thresholds ("Abs" and "Ess") in Figure 6 allow us to determine the absolute stability and stability, respectively, for all wavetrain solutions (2) to the lambdaomega equations. In Figure 6 we overlay plots for the amplitude selected by zero Dirichlet boundary conditions ("Dirichlet") using (4) and another wave selection scenario that will be described in the discussion ("Invasion"). This shows that the stability of wavetrain solutions selected by each boundary condition changes at critical values of $\omega_{1}$, although the critical values are different for the two different wave selection scenarios (other than when $\omega_{1}=0$ ).

Copyright (c) by SIAM. Unauthorized reproduction of this article is prohibited. 


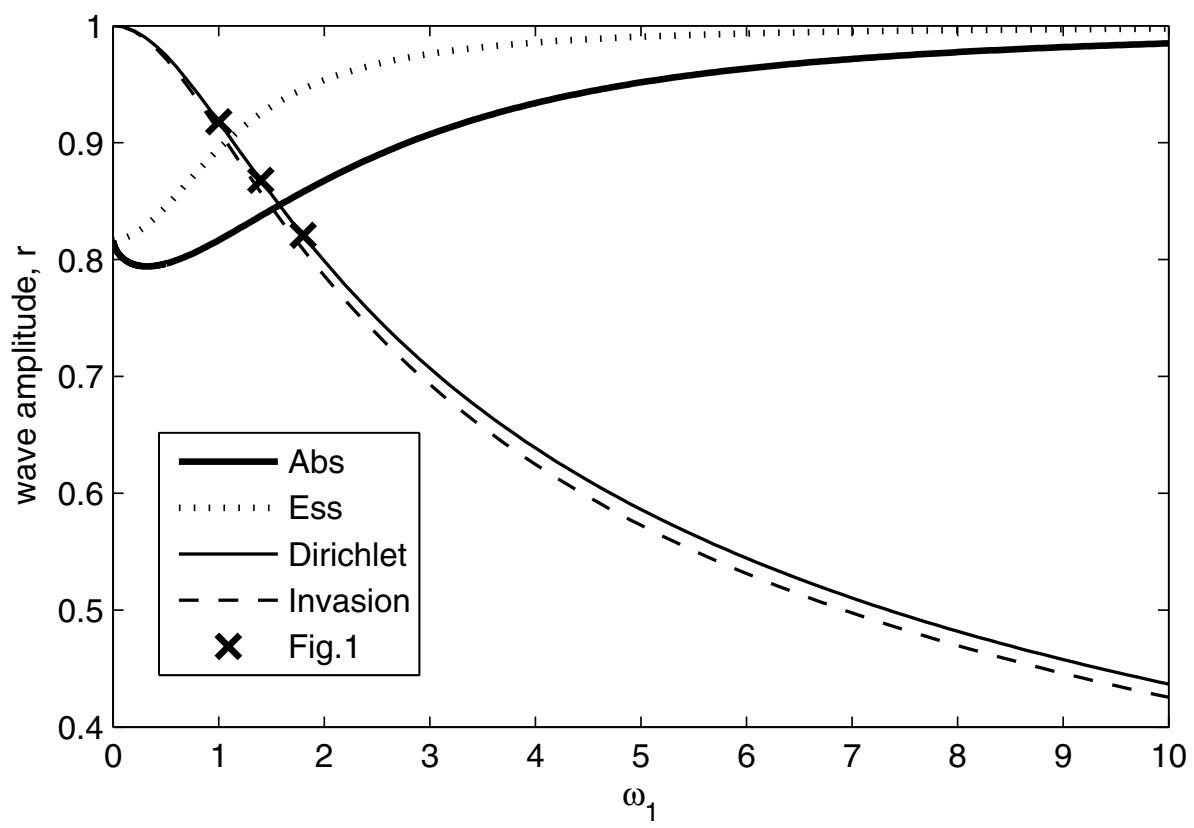

Figure 6. The relationship between $\omega_{1}$ and the critical amplitudes of wavetrain solutions to (1), above which waves are essentially or absolutely unstable (dotted and thick solid lines labeled "Ess" and "Abs," respectively). The critical amplitude for essential stability was determined using (9), and that for absolute stability was determined using numerical continuation, as described in section 4. We also overlay the amplitudes of wavetrains selected by zero Dirichlet boundary conditions (given by (4) and indicated by a thin solid line labeled "Dirichlet") and invasion (the dashed line labeled "Invasion"); the latter is simply a different wavetrain selection scenario (with selected wavetrain amplitude given by (16)) and is discussed in section 6 . The crosses indicate the values of $\omega_{1}$ used in Figure 1.

Figure 6 also shows that at $\omega_{1}=0$ wavetrains cannot be convectively unstable; rather, an instability is always absolute (due to the additional symmetry).

We used continuation in AUTO to obtain an accurate estimate for the absolute stability parameter threshold for waves selected by zero Dirichlet boundary conditions, i.e., $R=r_{d i r}$, where $r_{d i r}$ is given by (4). This is the point at which the thick solid line labeled "Abs" crosses the thin solid line labeled "Dirichlet" in Figure 6. To obtain our estimate we performed the same continuations as before but restricting the wavetrain amplitude, $R$, to be a solution to (4). We again used one of the complex conjugate branch points as a starting point, but this time we performed the continuation in $\omega_{1}$, getting AUTO to indicate the precise value of $\omega_{1}$ at which $\operatorname{Re}(\lambda)=0$. This gave the absolute stability threshold as $\omega_{1}<1.576465$, which corresponds to $r_{d i r}=0.846456$.

5. Source-sink solutions. When the value of the parameter $\omega_{1}$ implies that zero Dirichlet boundary conditions select a convectively unstable wave, the solution has the form of bands of wavetrains with alternating directions of propagation (Figure 1(c),(d)). These bands are separated by localized defects known as sources and sinks, with the asymptotic wavetrains 
propagating away from sources and towards sinks. ${ }^{1}$ Sources and sinks have been studied extensively in the complex Ginzburg-Landau equation; for reviews, see [56, 1, 55]. For a discussion in the context of general oscillatory reaction-diffusion systems, see [37].

The occurrence of the alternating direction wavetrain bands for values of $\omega_{1}$ for which the underlying wavetrain is convectively unstable (and thus absolutely stable) provides a clear illustration of the difference between convective and absolute stability. When the wavetrain is convectively unstable, perturbations of an appropriate form grow with time, moving through space as they grow. For the lambda-omega system (1), the perturbations are moving in the same direction as wave propagation (when $\omega_{0}>\omega_{1}>0$ ). For an infinite (or sufficiently large finite) expanse of the wavetrain, the perturbation can move and grow without constraint, destabilizing the solution. However, in the context of a source-sink pattern, the perturbation is convected as far as the nearest downstream sink, where it is completely absorbed (illustrated in Figure 7). In contrast, if the wavetrain were absolutely unstable, the perturbation would grow at the site of initial application, unchecked by any surrounding sources and sinks. The stability of solution structures composed of finite segments of convectively unstable solutions has been established in a number of other contexts; see, for example, the work of $[40,28,34]$ on pulses in reaction-diffusion systems. Very recently, Sandstede and Scheel [36] have proved that, for the type of solution we are describing, stability depends precisely on the absolute stability of the underlying wavetrain.

Results of Sandstede and Scheel [37, section 6.8] enable a detailed characterization of the repeating source-sink pattern seen in Figure 1(c),(d). Provided that the width of each wavetrain band is large (relative to the half life of the decay of the sources and sinks to their asymptotic wavetrains), the solution form differs only slightly from a repeating pattern of stationary sources and sinks. Specifically, the difference is $O\left(e^{-\delta L}\right)$ for some $\delta>0$ as the source-sink separation $L \rightarrow \infty$. The exponentially small differences between the solutions in simulations such as Figure 1(c),(d) and stationary sources/sinks have one particularly important implication: the sources and sinks in our solutions move, though at an extremely slow speed (illustrated in Figure 8).

6. Discussion. Our main focus in this study has been to derive the spectra of wavetrains in a reaction-diffusion system of "lambda-omega" type, and to assess their utility in predicting the dynamics emerging in partial differential equation simulations on large bounded domains with a zero Dirichlet condition at one boundary. Our results indicate that it is possible to predict the dynamics that emerge in numerical simulations, using the wave selection formula of [41] in combination with numerical calculation of the stability spectra of the selected wavetrain. This leads us to identify three regions of parameter space with qualitatively different dynamics: stable wave selection, which has been explained in detail elsewhere; convectively

\footnotetext{
${ }^{1}$ The distinction between sources and sinks is actually based on group velocity rather than phase velocity. These are usually defined in terms of the wavenumber $k$ and temporal frequency $\Omega(k)$ of a wavetrain family, so that the solution is a function of $k x-\Omega(k) t$. Then the phase velocity is $\Omega(k) / k$, while the group velocity is $d \Omega(k) / d k$. For the lambda-omega system (1), the wavetrain solution $(u, v)=R(\sin \theta, \cos \theta)$ with $\theta=$ $\left\{\left(\omega_{0}-\omega_{1} R^{2}\right) t \pm\left(1-R^{2}\right)^{1 / 2} x\right\}$ therefore has phase velocity $\mp\left(\omega_{0}-\omega_{1} R^{2}\right) /\left(1-R^{2}\right)^{1 / 2}$ and group velocity $\mp 2 \omega_{1}\left(1-R^{2}\right)^{1 / 2}$. Hence our assumptions $\omega_{0}>\omega_{1}>0$ imply that the phase and group velocities have the same sign, since $R^{2} \leq 1$. Note, however, that if one were to take $\omega_{0}$ negative with $-\omega_{0}>\omega_{1}>0$, the signs would be opposite, so that waves would propagate towards sinks and away from sources.
}

Copyright (c) by SIAM. Unauthorized reproduction of this article is prohibited. 


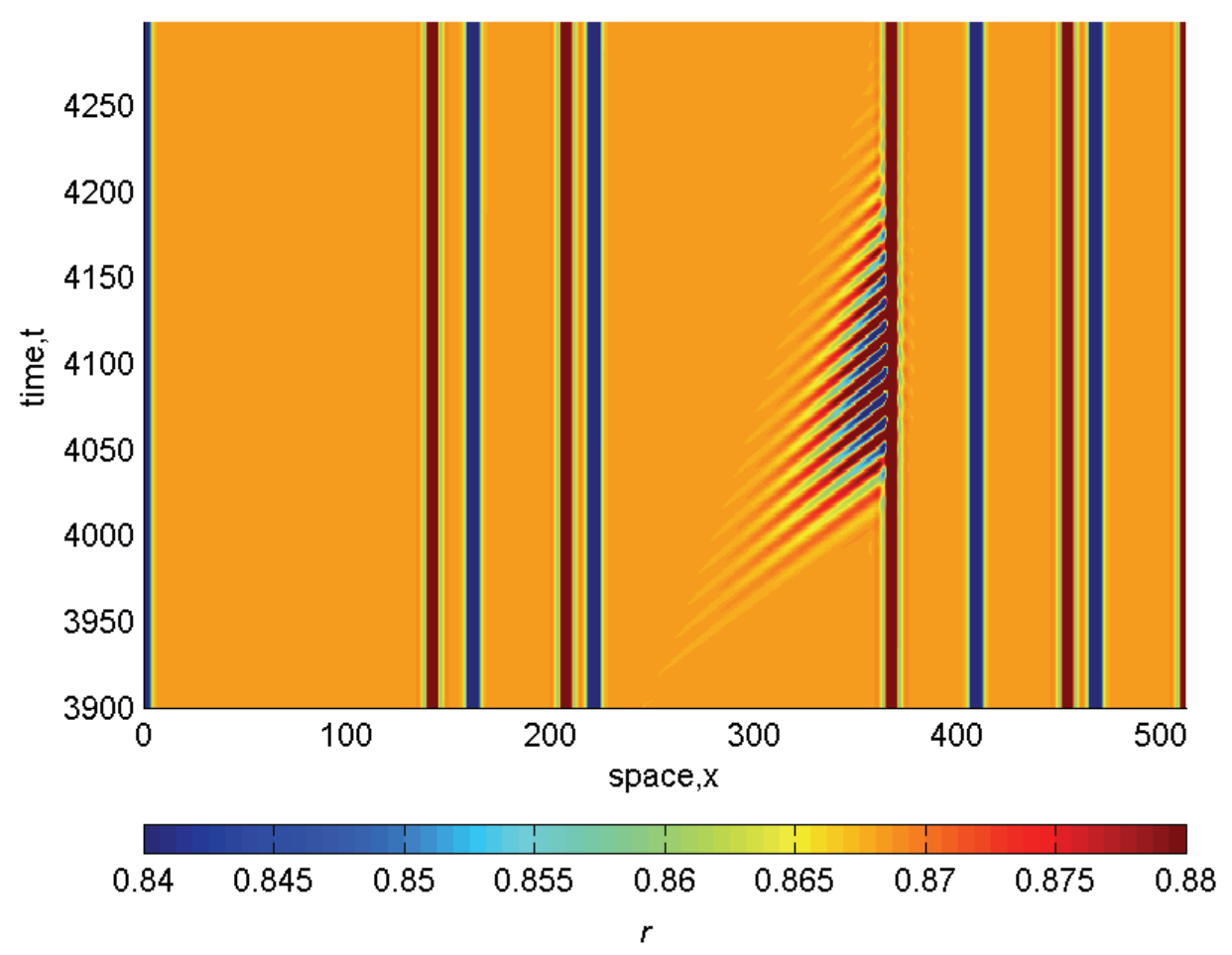

Figure 7. An illustration of the spatiotemporal evolution of a localized perturbation in the case of sourcesink dynamics. We solved (1) on $0<x<512$ subject to $u=v=0$ at $x=0$ and $u_{x}=v_{x}=0$ at $x=512$, up to time $t=3900$. The parameter $\omega_{1}=1.4$, so that the resulting wavetrain is convectively unstable, and a pattern of sources and sinks develops (see Figure 1(c),(d), which use the same parameter values). We then made a perturbation at one space point, increasing $u$ and $v$ by 0.1 at $x=194.82$, and then resumed the simulation up to $t=4300$. The perturbation is convected in the positive $x$ direction as far as the nearest sink, where it is completely absorbed.

unstable wave selection, in which we obtain bands of wavetrains separated by localized sources and sinks; and absolutely unstable wave selection, in which we observe irregular spatiotemporal dynamics.

Our subdivision of parameter space has important potential applications for predicting the spatiotemporal dynamics that will be observed in numerical simulations of other oscillatory reaction-diffusion systems, and in nature. For instance, a topical question for oscillatory ecological systems is whether or not they exhibit periodic traveling waves [5, 47]. Our results here suggest that the absolute stability threshold, rather than the stability threshold, may be more useful in predicting whether periodic traveling waves will be observed in the field. This is because the relevant ecological data is rarely, if ever, collected at sufficient spatial and temporal resolution to identify fine details of spatiotemporal dynamics such as source/sink defects. Furthermore, the usual size of habitats from which data is collected is far smaller than the domains considered here - a few spatial periods of the wavetrain would be typical. Our numerical simulations suggest that for such short domains there may be no qualitative difference between stable and convectively unstable wavetrains. 


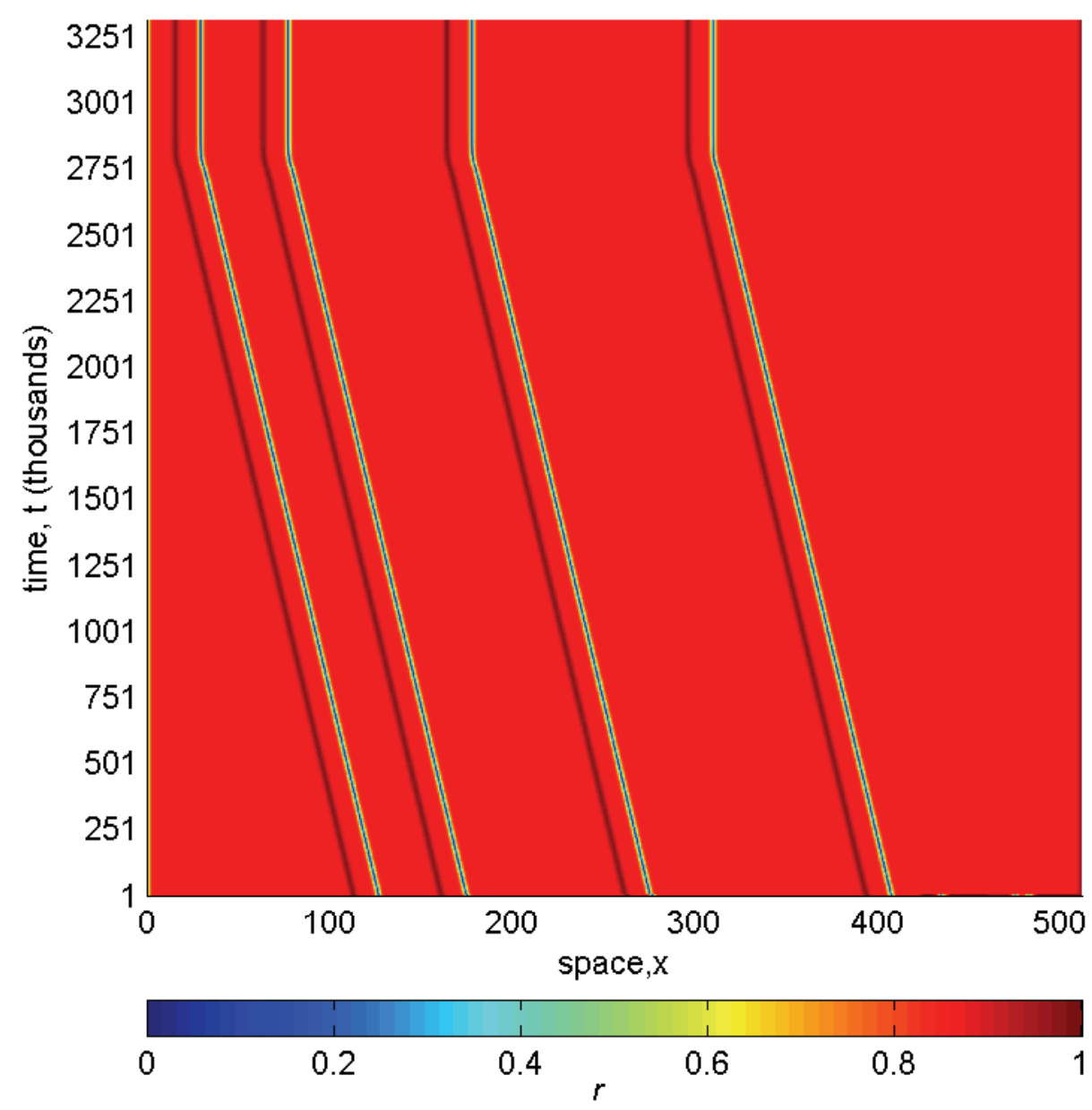

Figure 8. An illustration of the very slow spatial movement of the sources and sinks that occurs when the selected wavetrain is convectively unstable. We plot solution amplitude $r$ using a color scale. The parameter values and initial and boundary conditions are identical to those used in Figure 1(c),(d). Note the very long time scale of the plot (compare with the time scales in Figure 1(c)). Readers considering reproducing this figure should be aware that as a result of this long time scale, the simulation is very expensive in computer time: this run took 28 days on a Sun V20z computer with a 2.2 Ghz AMD Opteron processor.

On the mathematical side, we have shown rigorously that for (1), an instability of the absolute spectrum is always due to the crossing of a complex conjugate pair of branch points. In fact, we have proved this for a broader class of systems, and our approach may be extendable even further. In addition, we have shown that these branch points are exactly those determined by the pinching condition. We provide MATLAB code in the supplementary online material [49] to facilitate others in calculating stability thresholds via these critical branch points.

Our study has been aided by having an exact formula for the amplitude of selected waves for these particular boundary conditions. It would be interesting to compare these results with other wave selection scenarios, since one expects that different wave stabilities might be selected for the same values of $\omega_{1}$. The spatiotemporal dynamics of one other scenario,

Copyright (c) by SIAM. Unauthorized reproduction of this article is prohibited. 
invasion, has been studied in detail $[29,57,42]$. In this case, the boundary conditions are zero Neumann $\left(u_{x}=v_{x}=0\right)$ at both boundaries, with initial conditions consisting of a small localized perturbation to the unstable steady state $u=v=0$, adjacent to one boundary. Sherratt $[44,42,43]$ showed that such a perturbation induces a propagating invasion front, which for (1) always has a speed of 2 [57]. A fixed invasion speed is convenient for the purpose of simulations, as it makes it straightforward to calculate the solution time and domain size required to generate a given number of wavelengths.

The propagating invasion front selects the member of the periodic traveling wave family (2) with amplitude

$$
r_{i n v}=\left\{\frac{2}{\omega_{1}^{2}}\left[\sqrt{1+\omega_{1}^{2}}-1\right]\right\}^{1 / 2}
$$

(see $[29,57,42])$; this is slightly smaller than that selected by a zero Dirichlet boundary condition. The properties of the spatiotemporal dynamics that emerge behind the invasion front again depend on the stability of this wavetrain, which can be determined from Figure 6 . The wave is stable for $\omega_{1}<1.0714$ and absolutely stable for $\omega_{1}<1.5127$.

In Figure 9 we show the spatiotemporal dynamics arising from invasion for three different values of $\omega_{1}$. In all of our invasion simulations we assume that $u_{x}=v_{x}=0$ at both boundaries of the simulated domain and make an initial perturbation of $u=v=0.1$ on $0 \leq x<10$, with $u=v=0$ elsewhere.

As shown in [42], a wavetrain emerges behind the invasion front when $\omega_{1}$ is such that a stable member of the wavetrain family is selected by invasion (Figure $9(\mathrm{a}),(\mathrm{b})$ ). In this case the wavetrain travels in the opposite direction from the invasion front. When an unstable wavetrain is selected, we again see the wavetrain in a band behind the invasion front. However, this breaks up at a certain distance from the invasion front (Figure 9(c)-(f)). The existence of a propagating band of unstable waves behind an invasion front has been documented previously in numerical simulations of models for cyclic predator-prey systems $[45,46,31,30,23,19,20$, 24]. The wavetrain band becomes narrower as $\omega_{1}$ is increased, and persists as one crosses the threshold for absolute instability of the wavetrain; the behavior behind the band then changes from source-sink type (Figure 9(c),(d)) to more disordered dynamics (Figure 9(e),(f)). In a recent publication we present a method for calculating the dependence of the width of the wavetrain band on $\omega_{1}$ [48]. However, a more detailed investigation of the way in which the overall solution changes with $\omega_{1}$ is a natural area for future work.

Acknowledgments. The work in this paper has been greatly facilitated by very helpful discussions with Björn Sandstede (Brown University) and Leonid Brevdo (Louis Pasteur University). We thank the Microsoft Research Cambridge Tools and Technology Group (Computational Science) for technical assistance with the Lambda-Omega Equations Simulator, and two anonymous referees for helpful comments.

Copyright ( $\odot$ by SIAM. Unauthorized reproduction of this article is prohibited. 

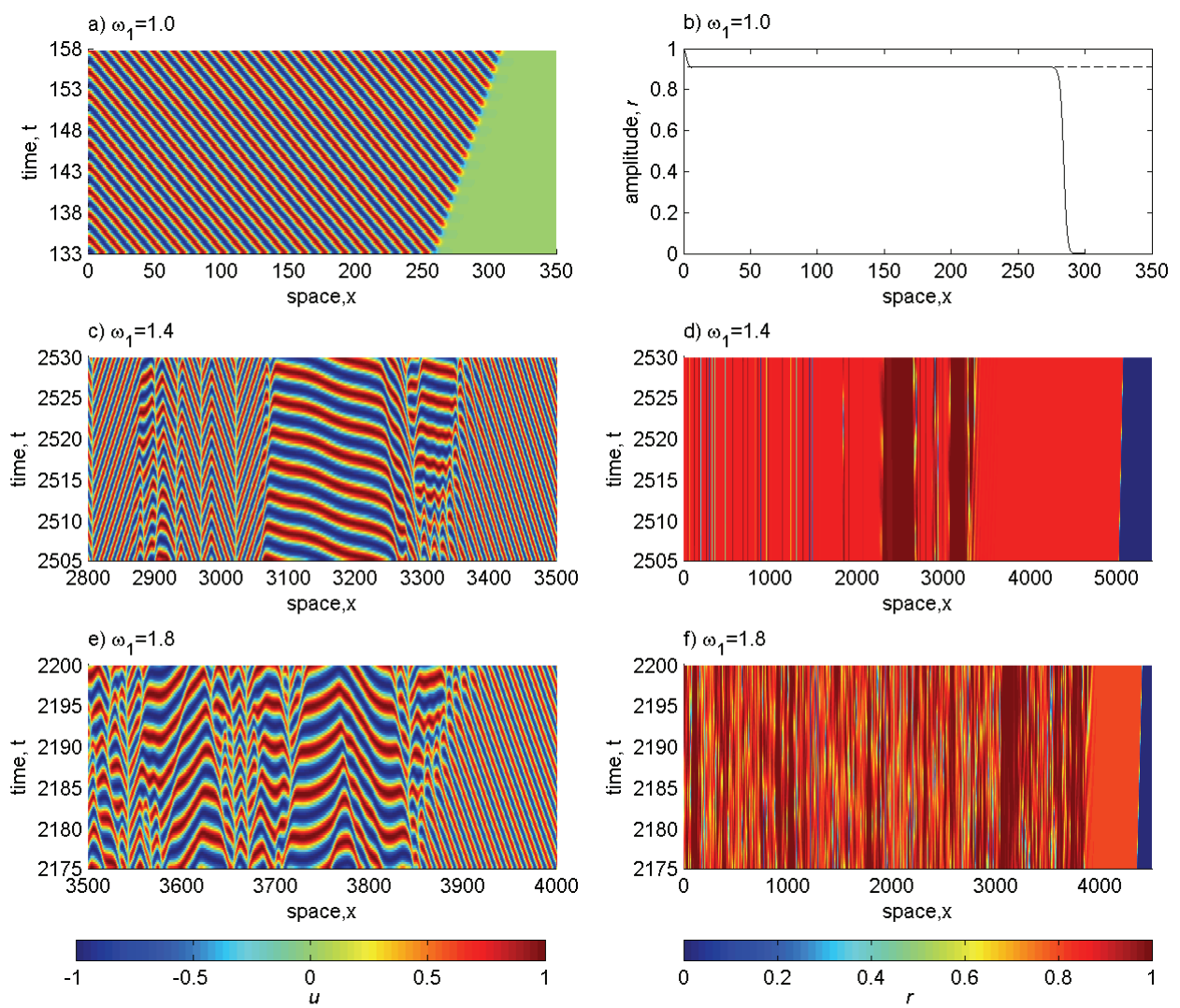

Figure 9. Example spatiotemporal dynamics for the lambda-omega equations (1) under an invasion scenario. Simulations started with $u=v=0$ throughout the domain except $u=v=0.1$ at $0<x<10$. We fix $u_{x}=v_{x}=0$ at both domain edges throughout the simulations. In all cases we show space-time plots for $u$ and the solution amplitude $r=\left(u^{2}+v^{2}\right)^{1 / 2}$. In (a),(b) we demonstrate the selection of a stable wave $\left(\omega_{1}=1.0\right)$. Parts $(\mathrm{c})-(\mathrm{f})$ show the spatiotemporal dynamics when the selected wave is convectively unstable $\left((\mathrm{c}),(\mathrm{d}): \omega_{1}=1.4\right)$ and absolutely unstable $\left((\mathrm{e}),(\mathrm{f}): \omega_{1}=1.8\right)$, respectively. In all cases $\omega_{0}=3.0$, and we plot $u$ and the solution amplitude $r=\sqrt{u^{2}+v^{2}}$. For clarity, we plot the long-term solution for $r$ as a function of space $x$ at one time point in (b), and show space-time plots in (a) and (c)-(f). We plot the solution across the whole domain in (a), (b), (d), and (f), but for only part of the domain in (c),(e).

\section{REFERENCES}

[1] I. S. Aranson And L. Kramer, The world of the complex Ginzburg-Landau equation, Rev. Modern Phys., 74 (2002), pp. 99-143.

[2] I. S. Aranson, L. Aranson, L. Kramer, And A. Weber, Stability limits of spirals and traveling waves in nonequilibrium media, Phys. Rev. A, 46 (1992), pp. R2992-R2995.

[3] J. F. G. Auchmuty And G. Nicoli, Bifurcation analysis of reaction-diffusion equations III. Chemical oscillations, Bull. Math. Biol., 38 (1976), pp. 325-350.

[4] N. BeKKi AND K. Nozaki, Formations of spatial patterns and holes in the generalised Ginzburg-Landau equation, Phys. Lett., 110A (1985), pp. 133-135.

[5] O. N. BJøRnstad, R. A. Ims, And X. Lambin, Spatial population dynamics: Analyzing patterns and processes of population synchrony, Trends Ecol. Evol., 14 (1999), pp. 427-432.

[6] J. F. Blowey and M. R. Garvie, A reaction-diffusion system of lambda-omega type. Part I: Mathematical analysis, European J. Appl. Math., 16 (2005), pp. 1-19.

Copyright ( ) by SIAM. Unauthorized reproduction of this article is prohibited. 
[7] L. Brevdo, P. Laure, F. Dias, and T. J. Bridges, Linear pulse structure and signalling in a film flow on an inclined plane, J. Fluid Mech., 396 (1999), pp. 37-71.

[8] L. Brevdo And T. J. Bridges, Absolute and convective instabilities of temporally oscillating flows, Z. Angew. Math. Phys., 48 (1997), pp. 290-309.

[9] L. Brevdo And T. J. Bridges, Absolute and convective instabilities of spatially periodic flows, Phil. Trans. R. Soc. Lond. A, 354 (1996), pp. 1027-1064.

[10] L. Brevdo, Convectively unstable wave packets in the Blasius boundary layer, Z. Angew. Math. Mech., 75 (1995), pp. 423-436.

[11] L. Brevdo, A study of absolute and convective instabilities with an application to the Eady model, Geophys. Astrophys. Fluid Dynam., 40 (1988), pp. 1-92.

[12] R. J. BRiggs, Electron-Stream Interaction with Plasmas, MIT Press, Cambridge, MA, 1964.

[13] J. M. Сномaz, Global instabilities in spatially developing flows: Non-normality and nonlinearity, in Annu. Rev. Fluid Mech. 37, Annual Reviews, Palo Alto, CA, 2005, pp. 357-392.

[14] M. Cross and P. C. Hohenberg, Pattern formation outside of equilibrium, Rev. Modern Phys., 65 (1993), pp. 851-1112.

[15] E. J. Doedel, Nonlinear numerics, J. Franklin Inst., 334 (1997), pp. 1049-1073.

[16] E. J. Doedel, AUTO, A program for the automatic bifurcation analysis of autonomous systems, Congr. Numer., 30 (1981), pp. 265-384.

[17] E. J. Doedel, W. Govaerts, Y. A. Kuznetsov, and A. Dhooge, Numerical continuation of branch points of equilibria and periodic orbits, in Modelling and Computations in Dynamical Systems, E. J. Doedel, E. J. Domokos, and I. G. Keverkidis, eds., World Scientific, River Edge, NJ, 2006, pp. $145-164$.

[18] G. B. Ermentrout, X. Chen, And Z. Chen, Transition fronts and localized structures in bistable reaction-diffusion equations, Phys. D, 108 (1997), pp. 147-167.

[19] M. R. GARVIE, Finite-difference schemes for reaction-diffusion equations modeling predator-prey interactions in MATLAB, Bull. Math. Biol., 69 (2007), pp. 931-956.

[20] M. R. Garvie And C. Trenchea, Finite element approximation of spatially extended predator-prey interactions with the Holling type II functional response, Numer. Math., 107 (2007), pp. 641-667.

[21] P. Huerre And P. A. Monkewitz, Local and global instabilities in spatially developing flows, in Annu. Rev. Fluid Mech. 22, Annual Reviews, Palo Alto, CA, 1990, pp. 473-537.

[22] N. Kopell and L. N. Howard, Plane wave solutions to reaction-diffusion equations, Stud. Appl. Math., 52 (1973), pp. 291-328.

[23] H. Malchow and S. V. Petrovski, Dynamical stabilization of an unstable equilibrium in chemical and biological systems, Math. Comput. Modelling, 36 (2002), pp. 307-319.

[24] H. Malchow, S. V. Petrovskit, and E. Venturino, Spatiotemporal Patterns in Ecology and Epidemiology: Theory, Models and Simulation, Chapman \& Hall/CRC Press, Boca Raton, FL, 2008.

[25] The Mathworks, Version 7.6.0.324, R2008a.

[26] J. D. Murray, Mathematical Biology II: Spatial Models and Biomedical Applications, Springer, New York, 2003.

[27] A. C. Newell, Envelope equations, in Nonlinear Wave Motion, Lectures in Appl. Math. 15, A. C. Newell, ed., American Mathematical Society, Providence, RI, 1974, pp. 157-163.

[28] S. NiI, The accumulation of eigenvalues in a stability problem, Phys. D, 142 (2000), pp. 70-86.

[29] K. Nozaki And N. BekKi, Exact solutions of the generalised Ginzburg-Landau equation, J. Phys. Soc. Japan, 53 (1984), pp. 1581-1582.

[30] S. V. Petrovskit, K. Kawasaki, F. Takasu, and N. Shigesada, Diffusive waves, dynamical stabilization and spatio-temporal chaos in a community of three competitive species, Japan J. Ind. Appl. Math., 18 (2001), pp. 459-481.

[31] S. V. Petrovskit And H. Malchow, Critical phenomena in plankton communities: Kiss model revisited, Nonlinear Anal. Real World Appl., 1 (2000), pp. 37-51.

[32] J. D. M. RADEMACHER, Geometric relations of absolute and essential spectra of wave trains, SIAM J. Appl. Dyn. Syst., 5 (2006), pp. 634-649.

[33] J. D. M. Rademacher, B. Sandstede, And A. Scheel, Computing absolute and essential spectra using continuation, Phys. D, 229 (2007), pp. 166-183.

[34] M. M. Romeo And C. K. R. T. Jones, Stability of neuronal pulses composed of concatenated unstable kinks, Phys. Rev. E, 63 (2001), paper 011904.

Copyright ( $\odot$ by SIAM. Unauthorized reproduction of this article is prohibited. 
[35] B. Sandstede, Stability of travelling waves, in Handbook of Dynamical Systems II, B. Fielder, ed., North-Holland, Amsterdam, 2002, pp. 983-1055.

[36] B. SAndstede And A. SCheEl, Interaction of Defects, manuscript, in preparation.

[37] B. Sandstede And A. Scheel, Defects in oscillatory media: Toward a classification, SIAM J. Appl. Dyn. Syst., 3 (2004), pp. 1-68.

[38] B. Sandstede And A. Scheel, Absolute and convective instabilities of waves on unbounded and large bounded domains, Phys. D, 145 (2000), pp. 233-277.

[39] B. Sandstede And A. Scheel, Absolute versus convective instability of spiral waves, Phys. Rev. E, 62 (2000), pp. 7708-7714.

[40] B. Sandstede And A. Scheel, Gluing unstable fronts and backs together can produce stable pulses, Nonlinearity, 13 (2000), pp. 1465-1482.

[41] J. A. Sherrate, Periodic travelling wave selection by Dirichlet boundary conditions in oscillatory reaction-diffusion systems, SIAM J. Appl. Math., 63 (2003), pp. 1520-1538.

[42] J. A. Sherrate, On the evolution of periodic plane waves in reaction-diffusion equations of $\lambda-\omega$ type, SIAM J. Appl. Math., 54 (1994), pp. 1374-1385.

[43] J. A. Sherratt, On the speed of amplitude transition waves in reaction diffusion equations of $\lambda-\omega$ type, IMA J. Appl. Math., 52 (1994), pp. 79-92.

[44] J. A. SherratT, The amplitude of periodic plane waves depends on initial conditions in a variety of $\lambda-\omega$ systems, Nonlinearity, 6 (1993), pp. 703-716.

[45] J. A. Sherratt, M. A. Lewis, And A. C. Fowler, Ecological chaos in the wake of invasion, Proc. Natl. Acad. Sci. USA, 92 (1995), pp. 2524-2528.

[46] J. A. Sherratt, B. T. Eagan, And M. A. Lewis, Oscillations and chaos behind predator-prey invasion: Mathematical artifact or ecological reality?, Phil. Trans. R. Soc. B, 352 (1997), pp. 21-38.

[47] J. A. Sherratt And M. J. Smith, Periodic travelling waves in cyclic populations: Field studies and reaction-diffusion models, J. R. Soc. Interface, 5 (2008), pp. 483-505.

[48] J. A. Sherratt, M. J. Smith, And J. D. M. RAdemacher, Locating the transition from periodic oscillations to spatiotemporal chaos in the wake of invasion, Proc. Natl. Acad. Sci. USA, 106 (2009), pp. 10890-10895.

[49] M. J. Smith, J. D. M. Rademacher, and J. A. Sherratt, Details on the computation of absolute spectra for periodic travelling waves in the Lambda-Omega equations using MATLAB and AUTO, online at http://research.microsoft.com/en-us/projects/loptw/tutorial.aspx.

[50] M. J. Smith, J. A. Sherratt, And N. J. Armstrong, The effects of obstacle size on periodic travelling waves in oscillatory reaction-diffusion equations, Proc. R. Soc. Lond. A, 464 (2008), pp. 365-390.

[51] S. A. Suslov, Convective and absolute instabilities in non-Boussinesq mixed convection, Theoret. Comp. Fluid Dynam., 21 (2007), pp. 271-290.

[52] S. A. SusLov, Numerical aspects of searching convective/absolute instability transition, J. Comput. Phys., 212 (2006), pp. 188-217.

[53] S. A. Suslov AND S. PAOlucci, Stability of non-Boussinesq convection via the complex Ginzburg-Landau model, Fluid Dyn. Res., 35 (2004), pp. 159-203.

[54] S. A. Suslov, Searching convective/absolute instability boundary for flows with fully numerical dispersion relation, Comput. Phys. Comm., 142 (2001), pp. 322-325.

[55] M. van HeCKe, Coherent and incoherent structures in systems described by the $1 D$ CGLE: Experiments and identification, Phys. D, 174 (2003), pp. 134-151.

[56] W. VAn SAArloos, Fronts, pulses, sources and sinks in generalized complex Ginzburg-Landau equations, Phys. D, 56 (1992), pp. 303-367.

[57] W. Van SaArloos, Front propagation into unstable states, Phys. Rep., 386 (2003), pp. 29-222.

[58] V. K. VANAG AND I. R. EPSTEIn, Localized patterns in reaction-diffusion systems, Chaos, 17 (2007), paper 037110.

[59] P. Wheeler and D. Barkley, Computation of spiral spectra, SIAM J. Appl. Dyn. Syst., 5 (2006), pp. $157-177$.

Copyright (C) by SIAM. Unauthorized reproduction of this article is prohibited. 